\title{
A quasi-linear time algorithm for computing modular polynomials in dimension 2
}

\author{
Enea Milio
}

\begin{abstract}
We propose to generalize the work of Régis Dupont for computing modular polynomials in dimension 2 to new invariants. We describe an algorithm to compute modular polynomials for invariants derived from theta constants and prove heuristically that this algorithm is quasi-linear in its output size. Some properties of the modular polynomials defined from quotients of theta constants are analyzed. We report on experiments with our implementation.
\end{abstract}

\section{Introduction}

An isogeny is a morphism between two abelian varieties that is surjective and has a finite kernel. It is an important notion for the theorical study of abelian varieties, but also for cryptographic applications because it allows one to transfer the discrete logarithm problem from a variety, where the problem is difficult, to an isogenous variety, where it may be easier.

The computation of an isogeny could mean several things: to be able to compute the isogenous variety, given a maximal isotropic subgroup of the $\ell$-torsion; to calculate the image of a point by an isogeny; and to check if two abelian varieties are isogenous and if so compute an isogeny. But what we are interested in is the computation of all the isogenous varieties (for a fixed degree) of a given variety and this can be done with modular polynomials.

These polynomials also have other applications. In dimension 1, they are key to the SchoofElkies-Atkin algorithm (SEA) for speeding up Schoof's algorithm for counting the number of points on an elliptic curve (see $[\mathbf{1 1}, \mathbf{4 0}]$ ), they are used for constructing elliptic curves with a known number of points by complex multiplication (see $[\mathbf{2}, \mathbf{1 5}, 43])$ and used for the computation of the endomorphism ring of elliptic curves (see [4]). They can be computed in quasi-linear time (see $[\mathbf{6}, \mathbf{1 2}]$ ).

In dimension 2 , these polynomials play the same role, but are harder to compute. They could also speed up the Chinese remainder theorem (CRT) algorithm (see [10]) to compute class fields of degree $4 \mathrm{CM}$-fields, which would lead to faster algorithms to construct cryptographically secure Jacobians of hyperelliptic curves.

An algorithm to compute the modular polynomials in dimension 2 was introduced by Régis Dupont (see [9]) in 2006. Using it, he managed to compute the polynomials parameterizing $(2,2)$-isogenies, but these polynomials are so big that he could not compute them for $(3,3)$ isogenies. This is due to the fact that he used Igusa invariants (see Definition 13).

In this article, we will present a generalization of his algorithm that allows one to compute modular polynomials with invariants $f_{1}, f_{2}, f_{3}$ derived from theta constants, for a congruence subgroup $\Gamma$ of the symplectic group $\Gamma_{2}$. We present results with Streng invariants (see Definition 28) and quotients of theta constants. The algorithm proceeds by evaluation/interpolation: without knowing the polynomials we are still able to evaluate them for any values and, if we do so for a sufficient number of values, we can interpolate to recover

Received 3 November 2014; revised 5 May 2015.

2010 Mathematics Subject Classification 11F46, 11 Y16 (primary).

This research was partially funded by ERC Starting Grant ANTICS 278537. 
the polynomials. The most important difference between this and the dimension 1 case is that we have to interpolate trivariate rational functions rather than univariate polynomials. This adds difficulties to the evaluation step; we need to choose the arguments in which to evaluate in a specific way. For this, we will see that we need to be able to find $\Omega$ modulo $\Gamma$ from $f_{1}(\Omega), f_{2}(\Omega)$ and $f_{3}(\Omega)$.

To do that, we deduce from $f_{1}(\Omega), f_{2}(\Omega)$ and $f_{3}(\Omega)$ the Igusa invariants $j_{1}(\Omega), j_{2}(\Omega)$, $j_{3}(\Omega)$ and then we apply Mestre's algorithm to obtain a hyperelliptic curve with these Igusa invariants. Using Thomae's formula, numerical integration and the Borchardt mean it is possible to obtain $\Omega$ modulo $\Gamma_{2}$, under Conjecture 23. Once we have $\Omega$ modulo $\Gamma_{2}$, we have to find $\Omega$ modulo $\Gamma$. This can be done, thanks to the functional equation of the theta constants of Proposition 8. Finally, we compute the products of Definition 27, which ends the evaluation step.

All the computation was done with multiprecision floating-point numbers. Explicit bounds on the size of the coefficients of the modular polynomials are unknown in dimension 2 (this is already a hard problem in dimension 1). Thus, our algorithm is heuristic. Using heuristics and Conjecture 23, we have shown that our algorithm is quasi-linear (Theorem 34). In practice, we may double the precision until we manage to find a sufficient precision to compute the polynomials. We emphasize the fact that the computations have been done at high precision so that we required a fast algorithm to compute the theta constants. This algorithm uses the Borchardt mean.

First, we applied Dupont's algorithm with Streng invariants instead of Igusa invariants to compute modular polynomials. The Streng invariants are equivalent to the Igusa ones in the sense that they describe the same moduli space up to birational equivalence (and indeed, there are formulae to switch from one to the other: see (5)). We managed to compute the modular polynomials parameterizing $(2,2)$ - and $(3,3)$-isogenies. The reason why this was possible is that the use of Streng invariants produces much smaller polynomials in terms of degree and size of the coefficients and thus the precision of the computation is smaller (as similarly noticed by Streng in [42] for the computation of class polynomials). For example, the modular polynomials for $p=2$ with Streng invariants fill 2.1 MB compared to $57 \mathrm{MB}$ with Igusa invariants.

Then, we applied our generalized algorithm to $b_{i}^{\prime}(\Omega)=\theta_{i}(\Omega / 2) / \theta_{0}(\Omega / 2)$ for $i=1,2,3$, which are modular functions for the group $\Gamma(2,4)$, and computed the modular polynomials with these invariants for $p=3, p=5$ and $p=7$. As these polynomials fill respectively $175 \mathrm{~KB}, 200 \mathrm{MB}$ and $29 \mathrm{~GB}$, we have no hope of computing the modular polynomials for larger $p$. The polynomials found are much smaller than those with Igusa or Streng invariants. For comparison, for $p=3$ they fill $175 \mathrm{~KB}$ while those with Streng invariants fill $890 \mathrm{MB}$. We explain this by the fact that these polynomials have symmetries (Theorem 44) and are sparse (Theorem 45).

The remainder of this article is organized as follows. In the first section, we recall the theory of abelian varieties, of theta constants and of modular polynomials. In the second section, we explain how to interpolate multivariate rational functions and we do a complexity analysis. This section is independent of the others. The algorithm to compute the modular polynomials is given in the third section and some applications of it are described in the fourth section. The fifth section is dedicated to a deeper examination of these polynomials (in particular the symmetries appearing and an interpretation of the denominators of the coefficients of these polynomials). In $\S 6$ we discuss our implementation of the algorithm and finally in $\S 7$ we give some examples of hyperelliptic curves over finite fields with isogenous Jacobians that we managed to compute using our modular polynomials.

\section{Theory of modular polynomials}

The Siegel upper half-space $\mathcal{H}_{g}$ for dimension $g$ is the set of $g \times g$ symmetric matrices over the complex numbers with positive definite imaginary part. It is a moduli space for principally 
polarized abelian varieties (see [3, Proposition 8.1.2]). Indeed, a principally polarized abelian variety is a torus $\mathbb{C}^{g} /\left(\Omega \mathbb{Z}^{2 g}+\mathbb{Z}^{2 g}\right)$ for $\Omega \in \mathcal{H}_{g}$ (which is called a period matrix).

Let $I_{g}$ denote the identity matrix of size $g$ and $J=\left(\begin{array}{cc}0 & I_{g} \\ -I_{g} & 0\end{array}\right)$. We define the symplectic group of dimension $2 g$ as $\mathrm{Sp}_{2 g}(\mathbb{Z})=\left\{\gamma \in \mathrm{Gl}_{2 g}(\mathbb{Z}):{ }^{t} \gamma J \gamma=J\right\}$. It can easily be shown that if $\gamma=\left(\begin{array}{cc}A & B \\ C & D\end{array}\right)$, then $\gamma \in \operatorname{Sp}_{2 g}(\mathbb{Z})$ if and only if the following three equalities are verified.

$$
\begin{aligned}
& { }^{t} A C={ }^{t} C A, \quad A^{t} B=B^{t} A, \\
& { }^{t} B D={ }^{t} D B, \quad \text { which are equivalent to } \quad D^{t} C=C^{t} D, \\
& { }^{t} D A-{ }^{t} B C=I_{g}, \quad A^{t} D-B{ }^{t} C=I_{g},
\end{aligned}
$$

because $\mathrm{Sp}_{2 g}(\mathbb{Z})$ is closed under transposition of matrices. Moreover, $\mathrm{Sp}_{2 g}(\mathbb{Z})$ acts (from the left) on $\mathcal{H}_{g}$ by $\left(\begin{array}{ll}A & B \\ C\end{array}\right) \Omega=(A \Omega+B)(C \Omega+D)^{-1}$, the matrix $C \Omega+D$ being invertible for $\left(\begin{array}{ll}A & B \\ C & D\end{array}\right) \in \mathrm{Sp}_{2 g}(\mathbb{Z})$ and $\Omega \in \mathcal{H}_{g}$.

The quotient space $\mathrm{Sp}_{2 g}(\mathbb{Z}) \backslash \mathcal{H}_{g}$ is a moduli space for isomorphism classes of principally polarized abelian varieties of dimension $g$ (see [3, Theorem 8.2.6]).

We define $\Gamma_{g}=\operatorname{Sp}_{2 g}(\mathbb{Z})$. Note that $-I_{2 g}$ acts trivially on $\mathcal{H}_{g}$, so that some authors prefer to consider the projective symplectic group.

Proposition 1. The group $\Gamma_{g}$ is generated by $J$ and the $g(g+1) / 2$ matrices

$$
M_{i, j}=\left(\begin{array}{cc}
I_{g} & m_{i, j} \\
0 & I_{g}
\end{array}\right),
$$

where $m_{i, j}$ is the matrix of size $g$ all entries of which are 0 except for those at $(i, j)$ and $(j, i)$, which are equal to 1 .

Proof. This is a direct consequence of [29, Proposition 6, pp. 41-42].

Let $\mathcal{F}_{g} \subseteq \mathcal{H}_{g}$ be such that $\Omega=\left(\Omega_{u, v}\right)_{u, v \in[1, g]}$ is in $\mathcal{F}_{g}$ if and only if $\Omega$ verifies:

(1) $\left|\Re\left(\Omega_{u, v}\right)\right| \leqslant \frac{1}{2}$ for each $u, v \in[1, g]$;

(2) the matrix $\Im(\Omega)$ is reduced in the sense of Minkowski (see [29, Chapter I.2] for the definition);

(3) for each $\left(\begin{array}{ll}A & B \\ C & D\end{array}\right) \in \Gamma_{g},|\operatorname{det}(C \Omega+D)| \geqslant 1$.

REMARK 2. The third point has to be verified, in principle, for each matrix of $\Gamma_{g}$. However, for the dimensions 1 and 2 this condition has to be verified only for a well-known finite set (of cardinality 1 in dimension 1 and 19 in dimension 2, see [29, Proposition 3, p. 33] and [19]).

The set $\mathcal{F}_{g}$ is a fundamental domain in the sense that for all $\Omega \in \mathcal{H}_{g}$, there exists $\gamma \in$ $\Gamma_{g} /\left\langle \pm l_{2 g}\right\rangle$ such that $\gamma \Omega \in \mathcal{F}_{g}$ and $\gamma$ is unique if $\gamma \Omega$ is an inner point of $\mathcal{F}_{g}$.

Definition 3. Let $\Gamma$ be a subgroup of finite index of $\Gamma_{g}$ and $k \in \mathbb{Z}$. A Siegel modular form of weight $k$ for $\Gamma$ is a function $f: \mathcal{H}_{g} \rightarrow \mathbb{C}$ such that:

(1) $f$ is holomorphic on $\mathcal{H}_{g}$;

(2) $\forall \gamma=\left(\begin{array}{cc}A & B \\ C & D\end{array}\right) \in \Gamma$ and $\Omega \in \mathcal{H}_{g}, f(\gamma \Omega)=\operatorname{det}(C \Omega+D)^{k} f(\Omega)$;

(3) in the case $g=1, f$ has to be holomorphic at the cusps (see, for example, [38, Definition 2.3.1] for the definition).

Definition 4. Let $\Gamma$ a subgroup of finite index of $\Gamma_{g}$. A function $f: \mathcal{H}_{g} \rightarrow \mathbb{C}$ is a Siegel modular function for $\Gamma$ if and only if there are two Siegel modular forms $f_{1}$ and $f_{2}$ for $\Gamma$ of the same weight and such that $f=f_{1} / f_{2}$. 
From another point of view, a complex torus is an abelian variety if and only if it can be embedded into a projective space. This embedding can be done using theta functions. We will only focus on the classical theta functions because they provide a projective coordinate system for the principally polarized abelian varieties and because these functions can easily be handled computationally.

Definition 5. Let $\Omega \in \mathcal{H}_{g}$ and let $z$ be a vector of $\mathbb{C}^{g}$. The Riemann theta function is the function

$$
\theta: \mathbb{C}^{g} \times \mathcal{H}_{g} \rightarrow \mathbb{C}, \quad(z, \Omega) \mapsto \sum_{n \in \mathbb{Z}^{g}} \exp \left(i \pi^{t} n \Omega n+2 i \pi^{t} n z\right)
$$

and, for $a, b \in \mathbb{Q}^{g}$, the (classical) theta function with characteristic $(a, b)$ is

$$
\begin{aligned}
\theta\left[\begin{array}{l}
a \\
b
\end{array}\right](z, \Omega) & =\sum_{n \in \mathbb{Z}^{g}} \exp \left(i \pi^{t}(n+a) \Omega(n+a)+2 i \pi^{t}(n+a)(z+b)\right) \\
& =\exp \left(i \pi^{t} a \Omega a+2 i \pi^{t} a(z+b)\right) \theta(z+\Omega a+b, \Omega) .
\end{aligned}
$$

These functions converge absolutely and uniformly on every compact subset of $\mathbb{C}^{g} \times \mathcal{H}_{g}$ due to the fact that the imaginary part of $\Omega$ is positive definite.

Proposition 6. Let $\Omega \in \mathcal{H}_{g}$ and $n \in \mathbb{N}$. The functions $f: \mathbb{C}^{g} \rightarrow \mathbb{C}$ satisfying for each $z \in \mathbb{C}^{g}$ and each $m^{\prime}, m^{\prime \prime} \in \mathbb{Z}^{g}$,

$$
f\left(z+\Omega m^{\prime}+m^{\prime \prime}\right)=f(z) \exp \left(-i \pi n^{t} m^{\prime} \Omega m^{\prime}-2 i \pi n^{t} z m^{\prime}\right)
$$

are said to be functions of level $n$. They form a vector space of dimension $n^{g}$ denoted by $R_{n}^{\Omega}$.

Proof. See [35, §II.1].

TheOREM 7 (Lefschetz). For $n \geqslant 3$, any linearly independent set of $k \geqslant n^{g}$ functions of level $n$ provides an embedding of $\mathbb{C}^{g} /\left(\Omega \mathbb{Z}^{2 g}+\mathbb{Z}^{2 g}\right)$ into $\mathbb{P}^{k-1}(\mathbb{C})$. For $n=2$, the functions of level 2 map only to $\left(\mathbb{C}^{g} /\left(\Omega \mathbb{Z}^{2 g}+\mathbb{Z}^{2 g}\right)\right) / \sim$, where $\sim$ is the equivalence relation such that $z \sim-z$.

Proof. See [34, p. 29].

Several bases for $R_{n}^{\Omega}$ are well known. We use $\mathscr{F}_{n}=\left\{\theta\left[\begin{array}{l}0 \\ b\end{array}\right](z, \Omega / n), b \in(1 / n) \mathbb{Z}^{g} / \mathbb{Z}^{g}\right\}$, and $\mathscr{F}_{(n, 1)^{n}}=\left\{\theta\left[\begin{array}{l}0 \\ b\end{array}\right](z, \Omega)^{n}, b \in(1 / n) \mathbb{Z}^{g} / \mathbb{Z}^{g}\right\}$. Theta functions have many properties. The following two are useful for an implementation of these functions. For a matrix $X$, denote by $X_{0}$ the vector composed of the diagonal entries of $X$.

Proposition 8. Let $\gamma=\left(\begin{array}{cc}A & B \\ C\end{array}\right) \in \Gamma_{g}, e^{\prime}=\frac{1}{2}\left({ }^{t} A C\right)_{0}$ and $e^{\prime \prime}=\frac{1}{2}\left({ }^{t} D B\right)_{0}$. Then, for all vectors $a, b$ in $\mathbb{Q}^{g}, z$ in $\mathbb{C}^{g}$ and $\Omega$ in $\mathcal{H}_{g}$ we have

$$
\begin{aligned}
\theta\left[\begin{array}{l}
a \\
b
\end{array}\right](\gamma z, \gamma \Omega)= & \zeta_{\gamma} \sqrt{\operatorname{det}(C \Omega+D)} \exp \left(i \pi^{t} z(C \Omega+D)^{-1} C z\right) \theta\left[{ }^{t} \gamma\left(\begin{array}{l}
a \\
b
\end{array}\right)+\left(\begin{array}{c}
e^{\prime} \\
e^{\prime \prime}
\end{array}\right)\right](z, \Omega) \\
& \times \exp \left(-i \pi^{t} a A^{t} B a\right) \exp \left(-i \pi^{t} b C^{t} D b\right) \exp \left(-2 i \pi^{t} a B^{t} C b\right) \\
& \times \exp \left(-2 i \pi^{t}\left({ }^{t} A a+{ }^{t} C b+e^{\prime}\right) e^{\prime \prime}\right),
\end{aligned}
$$

where $\zeta_{\gamma}$ is an eighth root of unity depending only on $\gamma$.

Proof. See [28, Chapter 5, Theorem 2] or [7, Proposition 3.1.24]. 
REMARK 9. The eighth root of unity and the square root do not depend on the characteristic. As we will always consider quotients of theta functions, we do not need to know the root and the determination of the square root.

Note that a matrix acts on the characteristic. The next proposition $[\mathbf{3 5}$, p. 123$]$ allows us to speak about permutations.

Proposition 10. For each $\alpha, \beta \in \mathbb{Z}^{g}$ and $a, b \in \mathbb{Q}^{g}, \theta\left[\begin{array}{c}a+\alpha \\ b+\beta\end{array}\right](z, \Omega)=\exp \left(2 i \pi{ }^{t} a \beta\right) \theta\left[\begin{array}{l}a \\ b\end{array}\right](z, \Omega)$.

The theta constants of level $n$ are the theta functions of level $n$ evaluated at $z=0$. In the following, we will focus on the theta constants of genus 2 with characteristic in $\left\{0, \frac{1}{2}\right\}^{2}$.

To simplify the notation we define for all $a=\left(\begin{array}{l}a_{0} \\ a_{1}\end{array}\right)$ and $b=\left(\begin{array}{l}b_{0} \\ b_{1}\end{array}\right)$ in $\{0,1\}^{2}$

$$
\theta_{b_{0}+2 b_{1}+4 a_{0}+8 a_{1}}(\Omega):=\theta_{a, b}(\Omega):=\theta\left(\begin{array}{l}
a / 2 \\
b / 2
\end{array}\right)(0, \Omega) .
$$

We have the property that $\theta_{a, b}(\Omega)=(-1)^{t}{ }^{a b} \theta_{a, b}(\Omega)$, so that of the sixteen theta constants, six are identically zero (we say that they are odd) and we denote $\mathcal{P}=\{0,1,2,3,4,6,8,9,12,15\}$ the subscripts of the even theta constants.

The next proposition [28, Chapter IV, Theorem 1] establishes a relationship between the $\theta_{i}^{2}(\Omega)$ for $i=0, \ldots, 15$ and the $\theta_{i}(\Omega / 2)$ for $i=0, \ldots, 3$.

Proposition 11 (Duplication formula). For all $a, b \in\{0,1\}^{2}$ and $\Omega \in \mathcal{H}_{2}$, we have

$$
\theta_{a, b}^{2}(\Omega)=\frac{1}{4} \sum_{b_{1}+b_{2} \equiv b \bmod 2}(-1)^{t} a b_{1} \theta_{0, b_{1}}(\Omega / 2) \theta_{0, b_{2}}(\Omega / 2) .
$$

Proposition 12. Let $\Omega \in \mathcal{H}_{2}$ and $\Omega^{\prime} \in \mathcal{F}_{2}$ be in the same class for the action of $\Gamma_{2}$. Then, either the matrix $\Omega^{\prime}$ is diagonal and exactly one of the even theta constants evaluated in $\Omega$ vanishes and at the same time $\theta_{15}\left(\Omega^{\prime}\right)=0$, or $\Omega^{\prime}$ is not diagonal and no even theta constant vanishes in $\Omega$ (nor in $\Omega^{\prime}$ ).

Proof. See [9, Proposition 6.5 and Corollary 6.1].

Let

$$
\begin{aligned}
h_{4} & =\sum_{i \in \mathcal{P}} \theta_{i}^{8}, \quad h_{6}=\sum_{60 \text { triples }(i, j, k) \in \mathcal{P}^{3}} \pm\left(\theta_{i} \theta_{j} \theta_{k}\right)^{4}, \\
h_{10} & =\prod_{i \in \mathcal{P}} \theta_{i}^{2}, \quad h_{12}=\sum_{15 \text { tuples }}(i, j, k, l, m, n) \in \mathcal{P}^{6} \\
h_{16} & =\frac{1}{3}\left(h_{12} \theta_{j} \theta_{k} \theta_{l} \theta_{l} \theta_{m} \theta_{n}\right)^{4}, \\
& \left.h_{6} h_{10}\right) .
\end{aligned}
$$

(See for example $[\mathbf{9}, \mathbf{4 2}, \mathbf{4 5}]$ for the exact definition.) The functional equation of Proposition 8 shows that $h_{i}$ is a Siegel modular form of weight $i$ for the group $\Gamma_{2}$.

Definition 13. We call Igusa invariants or $j$-invariants the functions $j_{1}, j_{2}, j_{3}$ defined by

$$
j_{1}:=\frac{h_{12}^{5}}{h_{10}^{6}}, \quad j_{2}:=\frac{h_{4} h_{12}^{3}}{h_{10}^{4}}, \quad j_{3}:=\frac{h_{16} h_{12}^{2}}{h_{10}^{4}} .
$$

Theorem 14. The field $K$ of Siegel modular functions in dimension 2 is $\mathbb{C}\left(j_{1}, j_{2}, j_{3}\right)$.

Proof. See [27]. 
Generically, by [26], two principally polarized abelian surfaces are isomorphic if and only if they have the same $j$-invariants.

Let $\Gamma$ be a subgroup of $\Gamma_{2}$ of index $k$. Denote the field of meromorphic functions of $\mathcal{H}_{2}$ invariant under the action of $\Gamma$ by $\mathbb{C}_{\Gamma}$ (it is the function field of $\Gamma \backslash \mathcal{H}_{2}$ ). In particular, $\mathbb{C}_{\Gamma_{2}}=K$. By [18], $\mathbb{C}_{\Gamma}$ is a finite algebraic extension of degree $k$ of $\mathbb{C}_{\Gamma_{2}}$.

Let $f$ be a modular function, $\gamma \in \Gamma_{2}$ and $p$ a prime number. We define the matrix $\gamma_{p}:=$ $\left(\begin{array}{cc}A & p B \\ C / p & D\end{array}\right)$ and the functions $f^{\gamma}, f_{p}$ and $f_{p}^{\gamma}$ from $\mathcal{H}_{2} \rightarrow \mathbb{C}$ by $f^{\gamma}(\Omega)=f(\gamma \Omega), f_{p}(\Omega)=f(p \Omega)$ and $f_{p}^{\gamma}(\Omega)=f(p \gamma \Omega)$ respectively. Let $\Gamma_{0}(p):=\left\{\left(\begin{array}{cc}A & B \\ C & D\end{array}\right) \in \Gamma_{2}: C \equiv 0 \bmod p\right\}$.

The three functions $j_{\ell, p}:=\left(j_{\ell}\right)_{p}$ are invariant under the group $\Gamma_{0}(p)$. Indeed, if $\gamma \in \Gamma_{0}(p)$, then $p \gamma \Omega=\gamma_{p}(p \Omega)$ so that $j_{\ell, p}^{\gamma}(\Omega)=j_{\ell}(p \gamma \Omega)=j_{\ell}\left(\gamma_{p}(p \Omega)\right)=j_{\ell}(p \Omega)=j_{\ell, p}(\Omega)$. In other words, $\Omega$ is equivalent to $\gamma \Omega$ for $\gamma \in \Gamma_{2}$, but that does not mean that $p \Omega$ is equivalent to $p \gamma \Omega$ : it is the case only if $\gamma$ is in $\Gamma_{0}(p)$.

Let $C_{p}$ be a set of representatives of the quotient $\Gamma_{2} / \Gamma_{0}(p)$. The period matrices of the $(p, p)$-isogenous varieties of a variety $\Omega$ are the $p \gamma \Omega$ for $\gamma \in C_{p}$ (by [5, Theorem 3.2]).

Proposition 10.1 of [9] gives $C_{p}$ for each $p$ and it tells us that $\left[\Gamma_{2}: \Gamma_{0}(p)\right]=p^{3}+p^{2}+p+1$.

Lemma 15. For a prime $p, \mathbb{C}_{\Gamma_{0}(p)}$ equals $K\left(j_{\ell, p}\right)$ for $\ell=1,2,3$.

Proof. See [5, Lemma 4.2].

Note that the functions $j_{\ell}$ have poles at $\Omega \in \mathcal{H}_{2}$ such that $h_{10}(\Omega)=0$. This happens when $\theta_{i}(\Omega)=0$ for some $i$. By Proposition 12, if $\Omega^{\prime} \in \mathcal{F}_{2}$ is equivalent to $\Omega$, then $\Omega^{\prime}$ is diagonal. We deduce that $\Omega$ corresponds to a product of elliptic curves. So the functions $j_{\ell, p}$ have poles at $\Omega \in \mathcal{H}_{2}$ corresponding to varieties that are $(p, p)$-isogenous to a product of elliptic curves.

Let $\Phi_{1, p}(X)=\prod_{\gamma \in C_{p}}\left(X-j_{1, p}^{\gamma}\right)$. It is the minimal polynomial of $j_{1, p}$ over $K$. As the functions $j_{2, p}$ and $j_{3, p}$ are contained in $K\left(j_{1, p}\right)=K\left[j_{1, p}\right]$ by Lemma 15 , we define $\Phi_{2, p}(X)$, and $\Phi_{3, p}(X)$ to be the monic polynomials in $K[X]$ of degree less than $\operatorname{deg}\left(\Phi_{1, p}(X)\right)$ satisfying $j_{2, p}=\Phi_{2, p}\left(j_{1, p}\right)$ and $j_{3, p}=\Phi_{3, p}\left(j_{1, p}\right)$.

Furthermore, we have for $\ell=2,3$ that $\Phi_{\ell, p}\left(j_{1, p}\right)=\Psi_{\ell, p}\left(j_{1, p}\right) / \Phi_{1, p}^{\prime}\left(j_{1, p}\right)$ where

$$
\Psi_{\ell, p}(X)=\sum_{\gamma \in C_{p}} j_{\ell, p}^{\gamma} \prod_{\gamma^{\prime} \in C_{p} \backslash\{\gamma\}}\left(X-j_{1, p}^{\gamma^{\prime}}\right) .
$$

Definition 16. Let $p$ be a prime number. We call $\Phi_{1, p}(X), \Psi_{2, p}(X)$ and $\Psi_{3, p}(X)$ the $(p, p)$ modular polynomials for $j_{1}, j_{2}$ and $j_{3}$.

For any prime $p$, the modular polynomials $\Phi_{1, p}(X), \Phi_{2, p}(X), \Phi_{3, p}(X)$ lie in the ring $\mathbb{Q}\left(j_{1}, j_{2}, j_{3}\right)[X]$ (see $\left[\mathbf{5}\right.$, Theorem 5.2]). This is also the case for $\Psi_{\ell, p}(X)$ for $\ell=2,3$ so that we will focus on $\Phi_{1, p}(X), \Psi_{2, p}(X)$ and $\Psi_{3, p}(X)$. The evaluation map $\mathbb{C}\left(j_{1}, j_{2}, j_{3}\right) \rightarrow \mathbb{C}$ sending $j_{i}$ to $j_{i}(\Omega)$ maps these polynomials to polynomials in $\mathbb{C}[X]$. The meaning of $\Phi_{1, p}(X)$ is that its roots evaluated at $\Omega \in \mathcal{H}_{2}$ are the $j_{1}$-invariants of the principally polarized abelian surfaces that are $(p, p)$-isogenous to the variety $\Omega$. Moreover, if $x$ is such a root, then $\left(x, \Phi_{2, p}(x), \Phi_{3, p}(x)\right)$ are the $j$-invariants of a principally polarized abelian surface $(p, p)$-isogenous to a variety with invariants $\left(j_{1}(\Omega), j_{2}(\Omega), j_{3}(\Omega)\right)$.

Denote the locus of all the principally polarized abelian surfaces which are $(p, p)$-isogenous to a product of elliptic curves by $\mathcal{L}_{p}$. This locus, $\mathcal{L}_{p}$, is a 2-dimensional algebraic subvariety of the 3 -dimensional moduli space $\Gamma_{2} \backslash \mathcal{H}_{2}$, and can be parameterized by an equation $L_{p}=0$ for a polynomial $L_{p}$ in $\mathbb{Q}\left[j_{1}, j_{2}, j_{3}\right]$.

Lemma 17. The denominators of the coefficients of $\Phi_{1, p}(X), \Psi_{2, p}(X)$ and $\Psi_{3, p}(X)$ are all divisible by the polynomial $L_{p}$. 
Proof. See [5, Lemma 6.2].

We are particularly interested in the denominators of the modular polynomials because they are the cause of many difficulties when computing these polynomials.

\section{Interpolation}

In this section, we explain how to interpolate multivariate polynomials and rational fractions, which will be needed to compute modular polynomials by evaluation and interpolation. The problem is the following: we assume that we have an algorithm $f$ such that for any $x_{1}, \ldots, x_{n} \in$ $\mathbb{C}$ it returns the value $P\left(x_{1}, \ldots, x_{n}\right)$ (or a floating-point approximation thereof), where $P$ is an unknown multivariate polynomial or rational fraction $P\left(X_{1}, \ldots, X_{n}\right)$ with complex coefficients. We want to find $P$.

We define $\mathcal{M}(d)$ as the time to multiply polynomials of degree less than or equal to $d$ with coefficients having $N$ bits and $\mathcal{M}^{\prime}(N)$ as the time complexity to multiply two integers of $N$ bits. By [25, Corollary 8.19], we have that $\mathcal{M}(d) \in O\left(d \log d \mathcal{M}^{\prime}(N)\right)$ if we use the fast Fourier transform (FFT) and if we assume that $N \in \Omega(\log d)$, which is necessary to distinguish between the different roots of unity used in the FFT. Moreover, $\mathcal{M}^{\prime}(N) \in O(N \log N \log \log N)$ (see $[41])$.

Following the basic idea of [9], we work out all the details and give a complexity analysis.

\subsection{Interpolation of a multivariate polynomial}

The problem of interpolating a univariate polynomial $P$ is well known and can be solved by Lagrange's or Newton's method, which need $\operatorname{deg}(P)+1$ evaluations. The complexity of fast interpolation is $O(\mathcal{M}(\operatorname{deg}(P)) \log (\operatorname{deg}(P)))$ (see [25, $\S 10$, Corollary 10.12]).

In the case of a bivariate polynomial $P(X, Y)$, we notice that it can be written as

$$
P(X, Y)=\sum_{i=0}^{d_{X}}\left(\sum_{j=0}^{d_{Y}} c_{i, j} Y^{j}\right) X^{i}=\sum_{i=0}^{d_{X}} c_{i}(Y) X^{i}
$$

We can compute $P(X, y)$ for a fixed $y$ by evaluating $P\left(x_{i}, y\right)$ for $i=1, \ldots, d_{X}+1$ and interpolating. The $\ell$ th coefficient of this polynomial is $c_{\ell}(y)$, which is a univariate polynomial. It can be obtained if one has computed $c_{\ell}\left(y_{j}\right)$ for $d_{Y}+1$ values of $y_{j}$.

Thus, to obtain $P(X, Y)$ we proceed as follows. For $j$ from 1 to $d_{Y}+1$, fix $y_{j}$ and choose $d_{X}+1$ values of $x_{i}$ (one may choose the same values for different $j$ ), then evaluate $P\left(x_{i}, y_{j}\right)$ and interpolate a univariate polynomial to find $P\left(X, y_{j}\right)$. Finally, for each $\ell=0, \ldots, d_{X}$, interpolate $c_{\ell}(Y)$. Hence, interpolating a bivariate polynomial needs $\left(d_{Y}+1\right)\left(d_{X}+1\right)$ evaluations and the complexity for the interpolation is

$$
\left(d_{Y}+1\right) O\left(\mathcal{M}\left(d_{X}\right) \log \left(d_{X}\right)\right)+\left(d_{X}+1\right) O\left(\mathcal{M}\left(d_{Y}\right) \log \left(d_{Y}\right)\right) \subseteq \tilde{O}\left(d_{X} d_{Y} N\right) .
$$

The interpolation of a trivariate polynomial can be done in a similar way. We write it as

$$
P(X, Y, Z)=\sum_{i=0}^{d_{X}}\left(\sum_{j=0}^{d_{Y}}\left(\sum_{k=0}^{d_{Z}} c_{i, j, k} Z^{k}\right) Y^{j}\right) X^{i}=\sum_{i=0}^{d_{X}}\left(\sum_{j=0}^{d_{Y}} c_{i, j}(Z) Y^{j}\right) X^{i}=\sum_{i=0}^{d_{X}} c_{i}(Y, Z) X^{i} .
$$

If, for fixed $y$ and $z$, we evaluate $P\left(x_{i}, y, z\right), i=1, \ldots, d_{X}+1$, and then interpolate, we obtain $P(X, y, z)$ and the $\ell$ th coefficient is $c_{\ell}(y, z)$. This gives us a method to evaluate $c_{\ell}(Y, Z)$ for different values and we can use what we said about bivariate polynomials to find $c_{\ell}(Y, Z)$. 
Thus, we proceed as follows. For $j$ from 1 to $d_{Y}+1$ and for $k$ from 1 to $d_{Z}+1$ we evaluate $P\left(x_{i}, y_{j}, z_{k}\right)$ for $d_{X}+1$ values of $x_{i}$ and then we do $\left(d_{Y}+1\right)\left(d_{Z}+1\right)$ interpolations to obtain all the $P\left(X, y_{j}, z_{k}\right)$. Each of the $d_{X}+1$ coefficients $c_{\ell}(Y, Z)$ is a bivariate polynomial and we have previously given the complexity to obtain it. All in all, we will do $\left(d_{X}+1\right)\left(d_{Y}+1\right)\left(d_{Z}+1\right)$ evaluations and the complexity for the interpolation is

$$
\begin{aligned}
& \left(d_{Y}+1\right)\left(d_{Z}+1\right) O\left(\mathcal{M}\left(d_{X}\right) \log \left(d_{X}\right)\right)+\left(d_{X}+1\right)\left(d_{Z}+1\right) O\left(\mathcal{M}\left(d_{Y}\right) \log \left(d_{Y}\right)\right) \\
& \quad+\left(d_{X}+1\right)\left(d_{Y}+1\right) O\left(\mathcal{M}\left(d_{Z}\right) \log \left(d_{Z}\right)\right) \subseteq \tilde{O}\left(d_{X} d_{Y} d_{Z} N\right) .
\end{aligned}
$$

We can generalize this improved algorithm recursively to the case of a polynomial in $n$ variables $X_{1}, \ldots, X_{n}$. It takes $\prod_{i=1}^{n}\left(d_{X_{i}}+1\right)$ evaluations. The complexity for the interpolation of a polynomial in $n$ variables is

$$
\sum_{i=1}^{n} \prod_{\substack{j=1 \\ j \neq i}}^{n}\left(d_{X_{j}}+1\right) O\left(\mathcal{M}\left(d_{X_{i}}\right) \log d_{X_{i}}\right) \subseteq \tilde{O}\left(\prod_{i=1}^{n} d_{X_{i}} N\right) .
$$

Note the symmetry, which means that the ordering of the variables does not matter.

\subsection{Interpolation of a multivariate rational fraction}

We begin with the univariate case: $F(X)=A(X) / B(X)$, with $A(X)=\sum_{i=0}^{d_{X}^{A}} A_{i} X^{i} \in \mathbb{C}[X]$ and $B(X)=\sum_{i=0}^{d_{X}^{B}} B_{i} X^{i} \in \mathbb{C}[X]$. We look for the solution with minimal degrees. Each pair $(A, B)$ is then defined only up to a multiplicative constant.

Let $n=d_{X}^{A}+d_{X}^{B}+1$. Writing $A(X)-F(X) B(X)=0$ induces us to proceed with linear algebra: it suffices to evaluate $F$ for $n+1$ values of $x_{i}$ and to find the coefficients $A_{i}$ and $B_{i}$ by solving the following linear system

$$
\left(\begin{array}{cccccccc}
1 & x_{1} & \ldots & x_{1}^{d_{X}^{A}} & -F\left(x_{1}\right) & -F\left(x_{1}\right) \cdot x_{1} & \ldots & -F\left(x_{1}\right) \cdot x_{1}^{d_{X}^{B}} \\
\vdots & \ddots & \vdots & \vdots & \vdots & \vdots & \ddots & \vdots \\
1 & x_{n} & \ldots & x_{n}^{d_{X}^{A}} & -F\left(x_{n}\right) & -F\left(x_{n}\right) \cdot x_{n} & \ldots & -F\left(x_{n}\right) \cdot x_{n}^{d_{X}^{B}}
\end{array}\right) \cdot\left(\begin{array}{c}
A_{0} \\
\vdots \\
A_{d_{X}^{A}} \\
B_{0} \\
\vdots \\
B_{d_{X}^{B}}
\end{array}\right)=\left(\begin{array}{c}
0 \\
\vdots \\
0
\end{array}\right) .
$$

This method is easy to implement, but its complexity is bad. Another solution involves using Cauchy interpolation (see $[\mathbf{2 5}, \S 5.8]$ ) with the fast Euclidean algorithm $[\mathbf{2 5}, \S 11]$, which produces an algorithm of complexity $O(\mathcal{M}(n) \log (n))$. The number of evaluations is $n$.

We explain it briefly. Let $k$ and $m$ be such that $\operatorname{deg} A<k$ and $\operatorname{deg} B \leqslant m-k$. Take $x_{1}, \ldots, x_{m} \in \mathbb{C}$ and $y_{i}=F\left(x_{i}\right)$ for $1 \leqslant i \leqslant m$. Let $f$ be an interpolating polynomial. We look at polynomials $r(X)$ and $t(X)$ such that for any $i, r\left(x_{i}\right)=t\left(x_{i}\right) F\left(x_{i}\right)$; this implies $\left(r\left(x_{i}\right)=t\left(x_{i}\right) y_{i}=t\left(x_{i}\right) f\left(x_{i}\right)\right.$ if and only if $r \equiv t f \bmod \left(X-x_{i}\right)$ for all $\left.i\right)$ and, by the Chinese remainder theorem, it is equivalent to ask that $r \equiv t f \bmod g$, where $g=\prod_{i=1}^{m}\left(X-x_{i}\right)$.

Then, we use the extended Euclidean algorithm on $g$ and $f$. Let $r_{j}, s_{j}, t_{j}$ be the $j$ th row of the algorithm, where $j$ is minimal such that $\operatorname{deg} r_{j}<k$ (that is, $r_{1}=g, r_{2}=f, s_{1}=1$, $s_{2}=0, t_{1}=0, t_{2}=1$ and $r_{\ell}=g s_{\ell}+f t_{\ell}$ for each row $\ell$ ). By [25, Corollary 5.18, §5.8], $r_{j}$ and $t_{j}$ verify $r_{j}\left(x_{i}\right)=t_{j}\left(x_{i}\right) y_{i}$ and $t_{j}\left(x_{i}\right) \neq 0$ for all $i$.

Thus, it suffices to compute this row to interpolate the fraction $F$. It is possible to compute a single row with the fast Euclidean algorithm. 
We study now the bivariate case $F(X, Y)=A(X, Y) / B(X, Y)$, with

$$
A(X, Y)=\sum_{i=0}^{d_{X}^{A}} \sum_{j=0}^{d_{Y}^{A}} c_{i, j}^{A} X^{i} Y^{j}=\sum_{i=0}^{d_{X}^{A}} c_{i}^{A}(Y) X^{i} \in \mathbb{C}[X, Y]
$$

and similarly for $B(X, Y)$. One could use linear algebra, but the complexity would be very bad. Thus, we would like to proceed as in the bivariate case for polynomials, namely by fixing values $y_{j}$ and computing the fractions $F\left(X, y_{j}\right)$ and then by interpolating the coefficients as polynomials in $Y$.

If $F(X, Y) \in \mathbb{Q}[X, Y]$, then for each rational fraction found, one can force the numerator and the denominator to have content 1 , but because of the multiplicative constant, this will not work, as shown by the next example.

EXAMPle 18. Assume that we are searching $F(X, Y)=\left(3 X^{2} Y^{2}+Y+2\right) /(3 X Y+3)$ and that we find $F(X, 1)=\left(X^{2}+1\right) /(X+1), F(X, 2)=\left(12 X^{2}+4\right) /(6 X+3), F(X, 3)=$ $\left(27 X^{2}+5\right) /(9 X+3)$ and also $F(X, 5)=\left(75 X^{2}+7\right) /(15 X+3)$. For the greater coefficient of the numerator $c_{2}^{A}(Y)$ : if we interpolate with $y_{i}=2,3,5$, which gives us $c_{2}^{A}\left(y_{i}\right)=12,27,75$, we find $3 Y^{2}$, which is correct; on the other hand, with $y_{i}=1,2,3$ giving $c_{2}\left(y_{i}\right)=1,12,27$, we obtain the polynomial $2 Y^{2}+5 Y-6$, which is completely wrong because of the simplification of the numerator and the denominator in the case $Y=1$.

REMARK 19. For some values of $Y$, there is a simplification by a polynomial; for example, $F(X,-5)=-5 X-1$. This is something that is noticed immediately because the degree of $X$ is smaller. We assume that every time it happens, we ignore the result.

In the above example, if we had fixed the coefficient of degree 0 of the denominator of the univariate rational fraction at some value ( 3 for instance), the simplification would not have been a problem. However, this is not true in general.

EXAMPLE 20. This time write

$$
F(X, 1)=\frac{X^{2}+1}{X+1}, \quad F(X, 2)=\frac{3 X^{2}+1}{\frac{3}{2} X+\frac{3}{4}}, \quad F(X, 3)=\frac{\frac{27}{5} X^{2}+1}{\frac{9}{5} X+\frac{3}{5}}, \ldots,
$$

where we always fix the coefficient of degree 0 of the numerator at 1 . Then, we deduce by interpolation that $c_{0}^{A}(Y)=1$, which is wrong. Indeed, when we divide by a constant to obtain the coefficient 1 , it is as if we had divided by $Y+2$.

Thus, the difficulty is that we have to normalize while being sure that the $i$ th coefficient of the numerator and the denominator of each fraction in $X$ comes from the evaluation of the same polynomial in $Y$.

This normalization is easy to obtain in the particular case where we already know one of the $c_{i}^{A}(Y)$ or $c_{i}^{B}(Y) \in \mathbb{C}[Y]$ (different from 0): we only have to multiply the solution by the constant, which gives us the correct evaluation for the known $c_{i}$. We can then obtain (using Cauchy interpolation) the fraction with $n\left(d_{Y}+1\right)$ evaluations and the complexity of the interpolation is

$$
\left(d_{Y}+1\right) O(\mathcal{M}(n) \log (n))+(n+1) O\left(\mathcal{M}\left(d_{Y}\right) \log \left(d_{Y}\right)\right) \subseteq \tilde{O}\left(d_{X} d_{Y} N\right)
$$

where $d_{X}=\max \left(d_{X}^{A}, d_{X}^{B}\right), d_{Y}=\max \left(d_{Y}^{A}, d_{Y}^{B}\right)$ and $n=d_{X}^{A}+d_{X}^{B}+1 \leqslant 2 d_{X}+1$. 
EXAmple 21. We continue the preceding example. Assume we know $c_{0}^{A}(Y)=Y+2$. We have $c_{0}^{A}(1)=3$ and instead of the fraction $\left(X^{2}+1\right) /(X+1)$ we take $\left(3 X^{2}+3\right) /(3 X+3)$. We also have $c_{0}^{A}(2)=4$ and we write $F(X, 2)=\left(12 X^{2}+4\right) /(6 X+3)$ and so on.

In general, an idea to avoid this difficulty is to consider the fraction $F^{\prime}(X, Y)=F(X, Y X)=$ $A^{\prime}(X, Y) / B^{\prime}(X, Y)$ because, in this case, $c_{0}^{B^{\prime}}(Y)$ is a constant. If it is not 0 , we can choose to fix it to be 1 and then the previous argument (one $c_{i}^{B^{\prime}}(Y)$ known) holds. Thus, we have $F(X, Y X)$ and we substitute $Y$ by $Y / X$ to obtain $F(X, Y)$. Since $d_{X}^{A^{\prime}}=d_{T}^{A}$ and $d_{X}^{B^{\prime}}=d_{T}^{B}$, where the subscript $T$ stands for the total degree, the complexity is $\tilde{O}\left(d_{T} d_{Y} N\right)$ (and $d_{T}=\max \left(d_{T}^{A}, d_{T}^{B}\right)$ ).

Note also that in the particular case where the coefficient of degree zero of $c_{0}^{B}(Y)$ is 0 , this method does not work. To overcome this difficulty, we can consider $F(X+r, Y+s)$ instead of $F(X, Y)$ for some values of $r$ and $s$ such that this coefficient will not be zero.

We study now the trivariate case. We want to interpolate $F(X, Y, Z)=A(X, Y, Z) / B(X, Y, Z)$ with $A(X, Y, Z), B(X, Y, Z)$ in $\mathbb{C}[X, Y, Z]$. Denote $d_{T}=\max \left(d_{T}^{A}, d_{T}^{B}\right)$ (and similarly for $d_{X}$, $d_{Y}$ and $\left.d_{Z}\right)$ and $n=d_{T}^{A}+d_{T}^{B}+1$. As in the bivariate case, we compute $F(X, X Y, X Z)$ and then substitute $Y$ by $Y / X$ and $Z$ by $Z / X$ to obtain $F(X, Y, Z)$. We will now explain how to compute $F(X, X Y, X Z)$ recursively.

(1) Suppose we are able to compute $F(X, X Y, z X)$ for a fixed $z \in \mathbb{C}$. Then we only need $d_{Z}+1$ evaluations in $z_{i}$ to interpolate (as polynomials) each coefficient in $Z$ and find $F(X, X Y, X Z)$. The number of coefficients is bounded above by $(n+1)\left(d_{Y}+1\right)$ so that the interpolation complexity for this step is $(n+1)\left(d_{Y}+1\right) O\left(\mathcal{M}\left(d_{Z}\right) \log \left(d_{Z}\right)\right)$.

(2) To obtain $F(X, X Y, z X)$ for a fixed $z$, it suffices to apply the interpolation algorithm in the bivariate case. We will do this step $d_{Z}+1$ times so that the complexity of the interpolation is $\left(d_{Z}+1\right)\left(\left(d_{Y}+1\right) O(\mathcal{M}(n) \log (n))+(n+1) O\left(\mathcal{M}\left(d_{Y}\right) \log \left(d_{Y}\right)\right)\right)$.

In doing this, the number of evaluations will be $n\left(d_{Y}+1\right)\left(d_{Z}+1\right)$ and the final interpolation complexity is $\tilde{O}\left(d_{T} d_{Y} d_{Z} N\right)$. (In the special case in which we already know one of the $c_{i}^{A}(Y, Z)$ or $c_{i}^{B}(Y, Z)$, the complexity will be $\left.\tilde{O}\left(d_{X} d_{Y} d_{Z} N\right)\right)$.

An improvement of this algorithm is obtained by noting that there is the possibility of substituting $Y$ by $Y / X$ in the second step to find $F(X, Y, z X)$ and to compute $F(X, Y, X Z)$ in the first one. Thus, the number of coefficients in the first step will be bounded above by $\left(n^{\prime}+1\right)\left(d_{Y}+1\right)$, where $n^{\prime}$ is $\operatorname{deg}_{X}^{A}(F(X, Y, z X))+\operatorname{deg}_{X}^{B}(F(X, Y, z X))+1$, which is $\leqslant n$ and allows the number of interpolations to be reduced. The complexity is then

$$
\begin{aligned}
& \left(d_{Y}+1\right)\left(d_{Z}+1\right) O(\mathcal{M}(n) \log (n))+(n+1)\left(d_{Z}+1\right) O\left(\mathcal{M}\left(d_{Y}\right) \log \left(d_{Y}\right)\right) \\
& \quad+\left(n^{\prime}+1\right)\left(d_{Y}+1\right) O\left(\mathcal{M}\left(d_{Z}\right) \log \left(d_{Z}\right)\right) \subseteq \tilde{O}\left(d_{T} d_{Y} d_{Z} N\right) .
\end{aligned}
$$

We can generalize this recursively to the case of a rational fraction $F$ with $m$ variables $X_{1}, \ldots, X_{m}$. We find that

$$
\prod_{i=2}^{m}\left(d_{X_{i}}+1\right) O(\mathcal{M}(n) \log (n))+\sum_{j=2}^{m} \prod_{\substack{i=2 \\ i \neq j}}^{m}\left(d_{X_{i}}+1\right) n(j) O\left(\mathcal{M}\left(d_{X_{j}}\right) \log \left(d_{X_{j}}\right)\right) \subseteq \tilde{O}\left(d_{T} \prod_{i=2}^{m} d_{X_{i}} N\right)
$$

where $n=d_{T}^{A}+d_{T}^{B}+1$ and $n(j)$ is one plus the degree in $X_{1}$ of the numerator plus the degree in $X_{1}$ of the denominator of $F\left(X_{1}, X_{2}, \ldots, X_{j-1}, X_{j} X_{1}, \ldots, X_{m} X_{1}\right)$.

Note that all these formulae for the complexity in the case of rational fractions are asymmetric, so that the choice of the order of the variables is important. The formulae suggest that it is preferable to take $X_{1}$ as the variable with the largest degree. In that case, $n \leqslant 6 d_{X_{1}}+1$ and the complexity of the interpolation is then $\tilde{O}\left(\prod_{i=1}^{m} d_{X_{i}} N\right)$. 


\section{Evaluation}

We have seen that the modular polynomials lie in the $\operatorname{ring} \mathbb{Q}\left(j_{1}, j_{2}, j_{3}\right)[X]$ so that we have to interpolate trivariate rational fractions in order to compute them. Using the method of interpolation of a rational fraction $F$ exposed in the preceding section requires its evaluation at the points $F\left(x_{i}, x_{i} y_{j}, x_{i} z_{k}\right)$, where there exists $\Omega \in \mathcal{H}_{2}$ such that $\left(j_{1}(\Omega), j_{2}(\Omega), j_{3}(\Omega)\right)=$ $\left(x_{i}, x_{i} y_{j}, x_{i} z_{k}\right)$. Here, we use the method presented in [9] to deduce a matrix $\Omega \in \mathcal{H}_{2}$ from its $j$-invariants and then we suggest a way to extend this algorithm to other invariants.

\subsection{Computing modular polynomials with the $j$-invariants}

In practice, the modular polynomials have large coefficients and degrees. We use floatingpoint multiprecision to compute them and the letter $N$ will denote this precision, which is measured in bits. We have an input $(x, y, z) \in \mathbb{C}^{3}$ and we are looking for $\Omega \in \mathcal{H}_{2}$ such that $\left(j_{1}(\Omega), j_{2}(\Omega), j_{3}(\Omega)\right)=(x, y, z)$.

The key to doing this is to look at the Borchardt mean. Let $\left(z_{k}\right)_{k \in\{1,2,3\}} \in \mathbb{C}^{3}$. We define the Borchardt sequence for $k \in\{1,2,3\}$ by

$$
u_{0}^{(0)}=1 \quad \text { and } \quad u_{k}^{(0)}=z_{k}
$$

and recursively for all $n \geqslant 0$

$$
u_{0}^{(n+1)}=\frac{1}{4} \sum_{k=0}^{3} u_{k}^{(n)} \quad \text { and } \quad u_{k}^{(n+1)}=\frac{1}{4} \sum_{k_{1}+k_{2} \equiv k \bmod 4} v_{k_{1}}^{(n)} v_{k_{2}}^{(n)},
$$

where $v_{0}^{(n)}$ is any square root of $u_{0}^{(n)}$ and $v_{k}^{(n)}=0$ if $v_{0}^{(n)}=0$ or $u_{k}^{(n)}=0$, otherwise $v_{k}^{(n)}$ is the square root of $u_{k}^{(n)}$ such that $\left|v_{0}^{(n)}-v_{k}^{(n)}\right| \leqslant\left|v_{0}^{(n)}+v_{k}^{(n)}\right|$ and with $\Im\left(v_{k}^{(n)} / v_{0}^{(n)}\right)>0$ if there is equality.

This sequence converges to a unique complex number called the Borchardt mean, denoted by $B_{2}\left(\left(z_{k}\right)_{k \in\{1,2,3\}}\right)$. Let $b_{i}(\Omega):=\theta_{i}^{2}(\Omega) / \theta_{0}^{2}(\Omega)$ for $1 \leqslant i \leqslant 15$. We have the following proposition.

Proposition $22\left[9\right.$, Proposition 9.1]. For all $\tau \in \mathcal{F}_{2}, B_{2}\left(b_{1}(\tau), b_{2}(\tau), b_{3}(\tau)\right)=1 / \theta_{0}^{2}(\tau)$.

Note that from this proposition and the ten even $b_{i}(\Omega)$, we can deduce all the $\theta_{i}^{2}(\Omega)=$ $b_{i}(\Omega) \theta_{0}^{2}(\Omega)$ at the working precision (with some loss of precision).

Conjecture 23 [9, Conjecture 9.1]. With the notation of Proposition 1 we have, for all $\tau \in \mathcal{F}_{2}$ and for all $\gamma \in\left\{\left(J M_{1,1}\right)^{2},\left(J M_{1,2}\right)^{2},\left(J M_{2,2}\right)^{2}\right\}: B_{2}\left(b_{1}(\gamma \tau), b_{2}(\gamma \tau), b_{3}(\gamma \tau)\right)=1 / \theta_{0}^{2}(\gamma \tau)$.

If this conjecture is true, it can easily be shown that for $\tau=\left(\begin{array}{cc}\tau_{1} & \tau_{3} \\ \tau_{3} & \tau_{2}\end{array}\right)$

$$
\begin{aligned}
\tau_{1} & =\frac{\imath}{\theta_{4}^{2}(\tau) B_{2}\left(\theta_{0}^{2}(\tau) / \theta_{4}^{2}(\tau), \theta_{6}^{2}(\tau) / \theta_{4}^{2}(\tau), \theta_{2}^{2}(\tau) / \theta_{4}^{2}(\tau)\right)}, \\
\tau_{2} & =\frac{\imath}{\theta_{8}^{2}(\tau) B_{2}\left(\theta_{9}^{2}(\tau) / \theta_{8}^{2}(\tau), \theta_{0}^{2}(\tau) / \theta_{8}^{2}(\tau), \theta_{1}^{2}(\tau) / \theta_{8}^{2}(\tau)\right)},
\end{aligned}
$$

and

$$
\tau_{3}^{2}-\tau_{1} \tau_{2}=\frac{1}{\theta_{0}^{2}(\tau) B_{2}\left(\theta_{8}^{2}(\tau) / \theta_{0}^{2}(\tau), \theta_{4}^{2}(\tau) / \theta_{0}^{2}(\tau), \theta_{12}^{2}(\tau) / \theta_{0}^{2}(\tau)\right)} .
$$

If $\tau$ is in the fundamental domain, the Minkowski reduction implies that $\Im\left(\tau_{3}\right) \geqslant 0$ which allows us to extract the correct square root and obtain $\tau$. Thus, it remains to show how to deduce, from $(x, y, z) \in \mathbb{C}^{3}$, the ten $b_{i}(\Omega)$, where $\Omega \in \mathcal{F}_{2}$ such that $\left(j_{1}(\Omega), j_{2}(\Omega), j_{3}(\Omega)\right)=$ $(x, y, z)$. This can be done in four steps. 
(1) The first one is to use Mestre's algorithm (see for example [32]) at precision $N$ to find a genus 2 curve $Y^{2}=f(X)$ over $\mathbb{C}$ with $f$ having degree 6 whose Igusa invariants are $(x, y, z)$.

(2) Once we have $f$, it is easy to deduce the set, $E$, of roots of $f$ at precision $N$ and from this set we use Thomae's formula (see $[44]$ or $[36, \S 8]$ ). Recall that this formula allows one to obtain the fourth power of the theta constants from an ordering of the roots of $f$ (which corresponds to a choice of the basis of the homology group of the Riemann surface of the hyperelliptic curve).

The problem here is that the functions $b_{i}$ are not invariant under the symplectic group $\Gamma_{2}$ (but are for a subgroup as we will see later). This means that for two matrices equivalent under the action of $\Gamma_{2}$ (namely that they have the same $j$-invariants), the evaluation of the $b_{i}$ in these matrices produces different results. Hence, the theta constants found with Thomae's formula give us $b_{i}^{2}(\gamma \Omega)$ for some unknown $\gamma \in \Gamma_{2}$.

(3) Now we use a numerical integration technique (see for example $[\mathbf{8}, \mathbf{2 4}, \mathbf{3 3}]$ ) at low precision $N^{\prime}$ with the same choice of the basis of the homology group to find the period matrix $\gamma \Omega$ that we reduce into the fundamental domain to obtain $\Omega$ at precision $N^{\prime}$ and $\gamma$. Compute $b_{i}(\Omega)$ at precision $N^{\prime}$ (with some algorithm to compute theta constants). We do not use a numerical integration technique at precision $N$ because it is too slow and it would increase the complexity of the algorithm.

(4) Using the functional equation of Proposition 8 on $b_{i}^{2}(\gamma \Omega)$ with the matrix $\gamma^{-1}$ allows one to obtain the $b_{i}^{2}(\Omega)$ at precision $N$ and knowing $b_{i}(\Omega)$ at precision $N^{\prime}$ is enough to deduce the correct square root and obtain $b_{i}(\Omega)$ at precision $N$.

Assumption 24. Note that we make the assumption that the numerical integration technique provides some $\gamma \Omega$ with $\gamma$ small enough such that it can be correctly reduced in the fundamental domain at the precision $N^{\prime}$.

Thus we obtain the following algorithm.

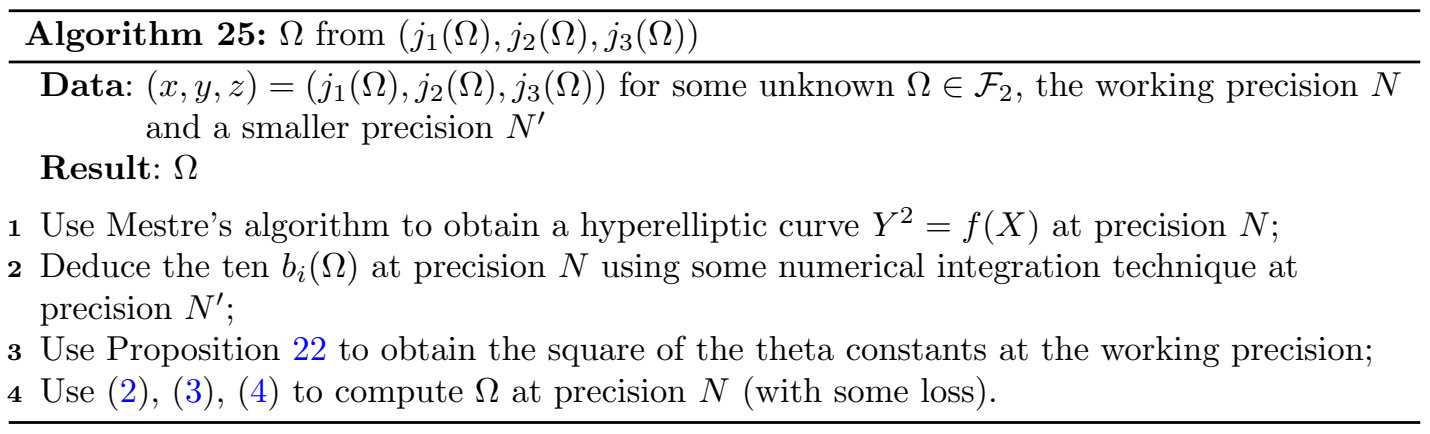

The second step is [9, Algorithm 12] and the third and fourth are Algorithm 13. They have complexity $O\left(\mathcal{M}^{\prime}(N)\right)$ and $O\left(\mathcal{M}^{\prime}(N) \log (N)\right.$ ) (where $\mathcal{M}^{\prime}(N)$ is the time complexity to multiply two integers of $N$ bits) so that the algorithm is in $\tilde{O}(N)$.

REMARK 26. Starting from $\left(1, b_{1}(\gamma \tau), b_{2}(\gamma \tau), b_{3}(\gamma \tau)\right)$ and an approximation of $\tau$, it is possible to compute a Borchardt sequence in which the square roots are chosen to be the $\theta_{i}\left(2^{n} \gamma \tau\right) / \theta_{0}(\gamma \tau)$ at each step. Using the duplication formula (Proposition 11), it can easily be proved that this will converge to $1 / \theta_{0}^{2}(\gamma \tau)$. Thus, it should be possible to not rely on the conjecture while maintaining the same complexity, as already stated in the first variant $[\mathbf{9}$, p. 200] and in the remark after [16, Theorem 12].

The conjecture has been tested and verified numerically by Dupont for many millions of random matrices. We emphasize that it is easy to test if the matrix $\Omega$ found at the end has the good $j$-invariants or not. 


\subsection{New invariants for the modular polynomials}

We begin by giving a generalization of the modular polynomials in order to have the possibility of using other invariants. In genus 1, this goes back to the works of Schläfli and Weber (see [39] and [12, $\S \S 4.2$ and 4.3]).

We consider only the congruence subgroups $\Gamma \subseteq \Gamma_{2}$, namely the groups with $\Gamma(n)=\{M \in$ $\left.\Gamma_{2}: M \equiv I_{4} \bmod n\right\} \subseteq \Gamma$ for some $n$. If $n$ is minimal with this property, we say that $n$ is the level of $\Gamma$. Let $\Gamma$ be a congruence subgroup and $f_{1}, f_{2}, f_{3}$ be three modular functions which are generators for the function field of $\Gamma \backslash \mathcal{H}_{2}$. Let $p$ be a prime number such that the level of $\Gamma$ is prime to $p$. Let $C_{p}$ be a set of representatives of $\Gamma /\left(\Gamma \cap \Gamma_{0}(p)\right)$.

Definition 27. The $(p, p)$-modular polynomials for these data are, for $\ell=2,3$,

$$
\Phi_{1, p}(X)=\prod_{\gamma \in C_{p}}\left(X-f_{1, p}^{\gamma}\right) \quad \text { and } \quad \Psi_{\ell, p}(X)=\sum_{\gamma \in C_{p}} f_{\ell, p}^{\gamma} \prod_{\gamma^{\prime} \in C_{p} \backslash\{\gamma\}}\left(X-f_{1, p}^{\gamma^{\prime}}\right) .
$$

We will sometimes write $\Phi_{1, p}\left(X, f_{1}, f_{2}, f_{3}\right)$ instead of $\Phi_{1, p}(X)$ and similarly for $\Psi_{\ell, p}(X)$. While the interpolation phase is still the same, the evaluation is slightly different: this time we have to find $\Omega \in \mathcal{H}_{2}$ from a triple $\left(x_{1}, x_{2}, x_{3}\right) \in \mathbb{C}$ such that $f_{i}(\Omega)=x_{i}$. Of course, this step depends on the three functions, but we will still give a general algorithm. On the other hand, the computation of $\Phi_{1, p}\left(X, f_{1}(\Omega), f_{2}(\Omega), f_{3}(\Omega)\right)$ and of $\Psi_{\ell, p}\left(X, f_{1}(\Omega), f_{2}(\Omega), f_{3}(\Omega)\right)$ for some $\Omega$ does not change. We can apply the same algorithm and they have the same complexity (except of course for the evaluation of the $f_{i}(p \gamma \Omega)$ ).

As in the dimension 1 case, we have tried to look at modular functions that would produce smaller modular polynomials than those with the $j$-invariants.

The first we tried are the invariants used by Streng in his thesis [42] to obtain smaller class polynomials. These invariants for $\Gamma_{2}$ are defined to have the minimal power of $h_{10}$ in the denominators.

Definition 28. We call Streng invariants the functions $i_{1}, i_{2}, i_{3}$ defined by

$$
i_{1}:=\frac{h_{4} h_{6}}{h_{10}}, \quad i_{2}:=\frac{h_{4}^{2} h_{12}}{h_{10}^{2}}, \quad i_{3}:=\frac{h_{4}^{5}}{h_{10}^{2}} .
$$

We will also say that these are $j$-invariants. The context will make it clear if we are speaking of Streng invariants or of Igusa invariants. This is justified by the next theorem. Note that it is easy to deduce from the Igusa invariants the Streng ones and vice versa. Indeed, we have

$$
i_{1}=\frac{j_{2}\left(j_{2}-3 j_{3}\right)}{2 j_{1}}, \quad i_{2}=\frac{j_{2}^{2}}{j_{1}}, \quad i_{3}=\frac{j_{2}^{5}}{j_{1}^{3}} \quad \text { and } \quad j_{1}=\frac{i_{2}^{5}}{i_{3}^{2}}, \quad j_{2}=\frac{i_{2}^{3}}{i_{3}}, \quad j_{3}=\frac{i_{2}^{2}\left(i_{2}-2 i_{1}\right)}{3 i_{3}} .
$$

Theorem 29. The field $K$ of Siegel modular functions in dimension 2 is $K=\mathbb{C}\left(j_{1}, j_{2}, j_{3}\right)=$ $\mathbb{C}\left(i_{1}, i_{2}, i_{3}\right)$.

Moreover, we also have the properties for $\ell=1,2,3$ and a prime $p$ that the three $i_{\ell, p}$ are invariants under the group $\Gamma_{0}(p)$ and that $\mathbb{C}_{\Gamma_{0}(p)}=K\left(i_{\ell, p}\right)$ (the proof is similar to the one for the Igusa invariants).

Thus, to compute the modular polynomials, the difference between this and the Igusa invariants is small: from $\left(i_{1}(\Omega), i_{2}(\Omega), i_{3}(\Omega)\right)$ to obtain $\Omega$, it is sufficient to use $(5)$ to deduce the triple $\left(j_{1}(\Omega), j_{2}(\Omega), j_{3}(\Omega)\right)$ and then to use Algorithm 25. The computation of the $i_{\ell}\left(p \gamma \Omega\right.$ ) (for a prime $p, \ell=1,2,3$ and $\gamma \in C_{p}$ ) is equivalent by (5) to the computation of the $j_{\ell}(p \gamma \Omega)$. 
The modular polynomials with Streng invariants are much smaller in terms of degree and precision of the coefficients than those with Igusa invariants so that the interpolation step can be done more rapidly and the number of times we use Algorithm 25 is also much smaller (see the next section).

Other invariants can be obtained by using the theta constants. This is motivated by the fact that the $j$-invariants are defined in terms of the theta constants.

Let $\Gamma(2,4)=\left\{\left(\begin{array}{cc}A & B \\ C & D\end{array}\right) \in \Gamma_{2}:\left(\begin{array}{cc}A & B \\ C & D\end{array}\right) \equiv I_{4} \bmod 2\right.$ and $\left.B_{0} \equiv C_{0} \equiv 0 \bmod 4\right\}$, which is a normal subgroup of $\Gamma_{2}$. It is well known that the $b_{i}(\Omega):=\theta_{i}^{2}(\Omega) / \theta_{0}^{2}(\Omega)$ are modular functions for the group $\Gamma(2,4)$. Actually, $\left[\mathbf{3 1}\right.$, Theorem 1] states that the field $\mathbb{C}_{\Gamma(2,4)}$ of modular functions belonging to $\Gamma(2,4)$ is $\mathbb{C}\left(b_{1}, \ldots, b_{15}\right)$.

Define for $i=1,2,3$ the functions $b_{i}^{\prime}(\Omega):=\theta_{i}(\Omega / 2) / \theta_{0}(\Omega / 2)$. From these three functions, it is easy to deduce the ten $b_{i}$ using the duplication formula (Proposition 11). The converse is also true because we have

$$
\begin{aligned}
& b_{1}^{\prime}=\left(b_{1}+b_{9}\right)\left(1+b_{4}+b_{8}+b_{12}\right)^{-1}, \\
& b_{2}^{\prime}=\left(b_{2}+b_{6}\right)\left(1+b_{4}+b_{8}+b_{12}\right)^{-1}, \\
& b_{3}^{\prime}=\left(b_{3}+b_{15}\right)\left(1+b_{4}+b_{8}+b_{12}\right)^{-1} .
\end{aligned}
$$

Thus, we consider the $b_{i}^{\prime}$ which allows us to handle three generators instead of ten.

Proposition 30. Let $p>2$ be a prime number. The classes of $\Gamma(2,4) /\left(\Gamma_{0}(p) \cap \Gamma(2,4)\right)$ are in bijection with the classes of $\Gamma_{2} / \Gamma_{0}(p)$.

Proof. Consider the map $\phi: \Gamma(2,4) \rightarrow \Gamma_{2} / \Gamma_{0}(p)$ with kernel $\Gamma_{0}(p) \cap \Gamma(2,4)$. The surjectivity comes from the Chinese remainder theorem and the fact that $\operatorname{Sp}(4, \mathbb{Z}) \rightarrow \operatorname{Sp}(4, \mathbb{Z} / 4 p \mathbb{Z})$ is surjective (the proof of which is analogous to $[\mathbf{3 0}, \S 6.1]$ ).

Proposition 31. For a prime $p>2, \mathbb{C}_{\Gamma(2,4) \cap \Gamma_{0}(p)}$ equals $\mathbb{C}_{\Gamma(2,4)}\left(b_{i, p}^{\prime}\right)$ for every $i=1,2,3$.

Proof. The proof is similar to that of [5, Theorem 4.2]. One has to use the isomorphism between $\Gamma(2,4) /(\Gamma(2,4) \cap \Gamma(p))$ and $\Gamma_{2} / \Gamma(p)$ which comes from the Chinese remainder theorem and the surjectivity of $\operatorname{Sp}(4, \mathbb{Z}) \rightarrow \operatorname{Sp}(4, \mathbb{Z} / 4 p \mathbb{Z})$.

Proposition 32. The modular polynomials for $b_{1}^{\prime}, b_{2}^{\prime}$ and $b_{3}^{\prime}$ lie in the ring $\mathbb{Q}\left(b_{1}^{\prime}, b_{2}^{\prime}, b_{3}^{\prime}\right)[X]$. More generally, it is also the case for any functions derived from the theta constants.

Proof. This comes from the fact that, for any $\Omega=\left(\begin{array}{ll}\Omega_{1} & \Omega_{2} \\ \Omega_{2} & \Omega_{3}\end{array}\right)$, the functions $b_{1}^{\prime}, b_{2}^{\prime}$ and $b_{3}^{\prime}$ have a Laurent series expansion in $q_{i}=\exp \left(2 \imath \pi \Omega_{i}\right)$ with rational coefficients. We conclude with a similar proof of [5, Theorem 5.2].

We want to deduce $\Omega$ from $\left(b_{1}^{\prime}(\Omega), b_{2}^{\prime}(\Omega), b_{3}^{\prime}(\Omega)\right)=\left(x_{1}, x_{2}, x_{3}\right)$. The first thing to do is to deduce from $\left(x_{1}, x_{2}, x_{3}\right)$ the Igusa invariants of $\Omega$. This can be done easily by calculating the ten $b_{i}(\Omega)$ from the three $x_{i}$ with the duplication formula and then by using the definition of the $j$-invariants (see Definition 13). We then execute Algorithm 25 to deduce a period matrix $\Omega^{\prime} \in \mathcal{H}_{2}$ from the $j$-invariants. Unfortunately, this $\Omega^{\prime}$ is equivalent to $\Omega$ in the sense that they have the same $j$-invariants, but this does not imply that $b_{i}^{\prime}\left(\Omega^{\prime}\right)=x_{i}$ because the functions $b_{i}$ are invariants for the group $\Gamma(2,4)$ (and not $\Gamma_{2}$ ).

To overcome this difficulty, we have to consider the cosets of $\Gamma_{2} / \Gamma(2,4)$. To find the good $\Omega$ modulo $\Gamma(2,4)$, we can take all the representatives $\gamma$ of this quotient and evaluate the three $b_{i}^{\prime}\left(\gamma \Omega^{\prime}\right)$ at low precision. The triple nearest to $\left(x_{1}, x_{2}, x_{3}\right)$ gives a matrix $\gamma^{\prime}$ and then 
we use the functional equation of Proposition 8 to obtain $b_{i}^{\prime}\left(\gamma^{\prime} \Omega^{\prime}\right)=b_{i}^{\prime}(\Omega)$ at the working precision $N$. But this index is large so that this method is slow. Indeed, $\left[\Gamma_{2}: \Gamma(2,4)\right]=11520$. The next method we propose is faster.

Another solution consists of precomputing the action (permutations and constants) of the representatives of $\Gamma_{2} / \Gamma(2,4)$ using the functional equation, comparing the three $b_{i}^{\prime}(\Omega)$ with the three $b_{i}^{\prime}\left(\Omega^{\prime}\right)$ to deduce the action, and finally to find a representative $\gamma$ in the precalculation which gives us $\gamma \Omega^{\prime}=\Omega$. The time spent for each $\gamma$ is thereby negligible.

More precisely, we know the three $b_{i}^{\prime}(\Omega)$ and thus the ten even $b_{i}(\Omega)$ (recall that it is equivalent by (6) to have the three $b_{i}^{\prime}$ ) and $\Omega^{\prime}$ (at the working precision). We compute the ten $b_{i}\left(\Omega^{\prime}\right)$ and we are looking for $\gamma$ such that $\gamma \Omega^{\prime}=\Omega$. Use the functional equation to obtain $\theta_{k}^{2}\left(\gamma \Omega^{\prime}\right)=\zeta_{\gamma}^{2} \operatorname{det}(\ldots) \imath^{\epsilon(\gamma, k)} \theta_{\ell}^{2}\left(\Omega^{\prime}\right)$ with $\epsilon(\gamma, k) \in\{0,1,2,3\}$. As the $b_{i}$ are quotients of theta constants, we can already forget about $\zeta_{\gamma}^{2}$ and $\operatorname{det}(\ldots)$. We will say in this case that $k$ is sent to $\ell$ by the action of $\gamma$. If 0 is sent to 0 , then the sets $A$ of the ten $b_{i}(\Omega)=b_{i}\left(\gamma \Omega^{\prime}\right)$ and $B$ of the ten $b_{i}\left(\Omega^{\prime}\right)$ are equal up to permutation and fourth roots of unity. It is easy to compare these two sets to deduce the action of the matrix $\gamma$. But, the difficulty is that 0 is not always sent to 0 and thus $b_{i}\left(\gamma \Omega^{\prime}\right)$ cannot be written as a root of unity times $b_{j}\left(\Omega^{\prime}\right)$ (if $i$ is sent to $j$ ), but as a root times a quotient of squares of theta constants evaluated at $\Omega^{\prime}$. However, note that there is a $c$ such that $c$ is sent to 0 and a $d$ such that 0 is sent to $d$. Thus, we have $b_{c}\left(\gamma \Omega^{\prime}\right)=$ $\imath^{\epsilon(\gamma, c)-\epsilon(\gamma, 0)} b_{d}\left(\Omega^{\prime}\right)^{-1}$ and comparing $A$ with $B$ up to a fourth root of unity it is possible to find $b_{d}\left(\Omega^{\prime}\right)$. Then we have $b_{k}\left(\gamma \Omega^{\prime}\right)=\imath^{\epsilon(\gamma, k)-\epsilon(\gamma, 0)} \theta_{\ell}^{2}\left(\Omega^{\prime}\right) / \theta_{d}^{2}\left(\Omega^{\prime}\right)=\imath^{\epsilon(\gamma, k)-\epsilon(\gamma, 0)} b_{\ell}\left(\Omega^{\prime}\right) b_{d}\left(\Omega^{\prime}\right)^{-1}$ and it is enough to multiply the set $A$ by $b_{d}\left(\Omega^{\prime}\right)^{-1}$ and compare it to $B$ to deduce the action of $\gamma$.

This method can also be used to modify Step 2 of Algorithm 25. Indeed, in the case where we cannot choose the basis of the homology group for the numerical integration, we obtain the period matrix $\Omega$ at low precision, but we do not know the matrix $\gamma$ such that $\gamma \Omega$ is the period matrix coming from Thomae's formula. As explained above, by comparing $b_{i}(\Omega)$ at low precision and $b_{i}(\gamma \Omega)$ at the working precision, we can still deduce $b_{i}(\Omega)$ at the working precision.

This is what we did in practice. We used the code of Pascal Molin (see [33]) for the numerical integration technique and we noticed that, given the roots of a polynomial of degree 6 , it returns a period matrix of the form $\gamma^{\prime \prime} \Omega^{\prime \prime}$, where $\Omega^{\prime \prime} \in \mathcal{F}_{2}$ and $\gamma^{\prime \prime}$ always seems to have $-1,0,1$ as coefficients. Thus, we never had any problem with the reduction in the fundamental domain of $\gamma^{\prime \prime} \Omega^{\prime \prime}$ (recall Assumption 24).

\subsection{Complexity analysis}

Let $f_{1}, f_{2}, f_{3}$ be three modular functions for a congruence subgroup $\Gamma$ of $\Gamma_{2}$ generating the function field of $\Gamma$. Let $p$ be a prime number which is prime to the level of $\Gamma$ and $C_{p}$ be a set of representatives of $\Gamma /\left(\Gamma \cap \Gamma_{0}(p)\right)$. We have explained in the preceding section how to find $\Omega$ from $\left(f_{1}(\Omega), f_{2}(\Omega), f_{3}(\Omega)\right)$ and then we have to evaluate the modular polynomials (see Definition 27) for a prime $p$ at $\Omega$, which means we have to compute

$$
\Phi_{1, p}\left(X, f_{1}(\Omega), f_{2}(\Omega), f_{3}(\Omega)\right) \quad \text { and } \quad \Psi_{\ell, p}\left(X, f_{1}(\Omega), f_{2}(\Omega), f_{3}(\Omega)\right)
$$

for $\ell=2,3$ (and each coefficient of these polynomials is the evaluation at $\Omega$ of a trivariate rational fraction in $f_{1}, f_{2}, f_{3}$ that we have to interpolate). To do that, we first compute $f_{\ell, p}^{\gamma}(\Omega)$ for all $\gamma \in C_{p}$ and $\ell=1,2,3$. Let $q=p^{3}+p^{2}+p+1$ the degree of $\Phi_{1, p}(X)$. The evaluation of $\Phi_{1, p}(X)$ at $\Omega$ can be obtained in $O(\mathcal{M}(q) \log q)$ using a subproduct tree (see [25, $\left.\S 10.1\right]$ ). The other two polynomials can be obtained with the same complexity using fast interpolation (see $[\mathbf{2 5}, \S 10.2])$. 
We summarize what we have explained by means of the following algorithm.

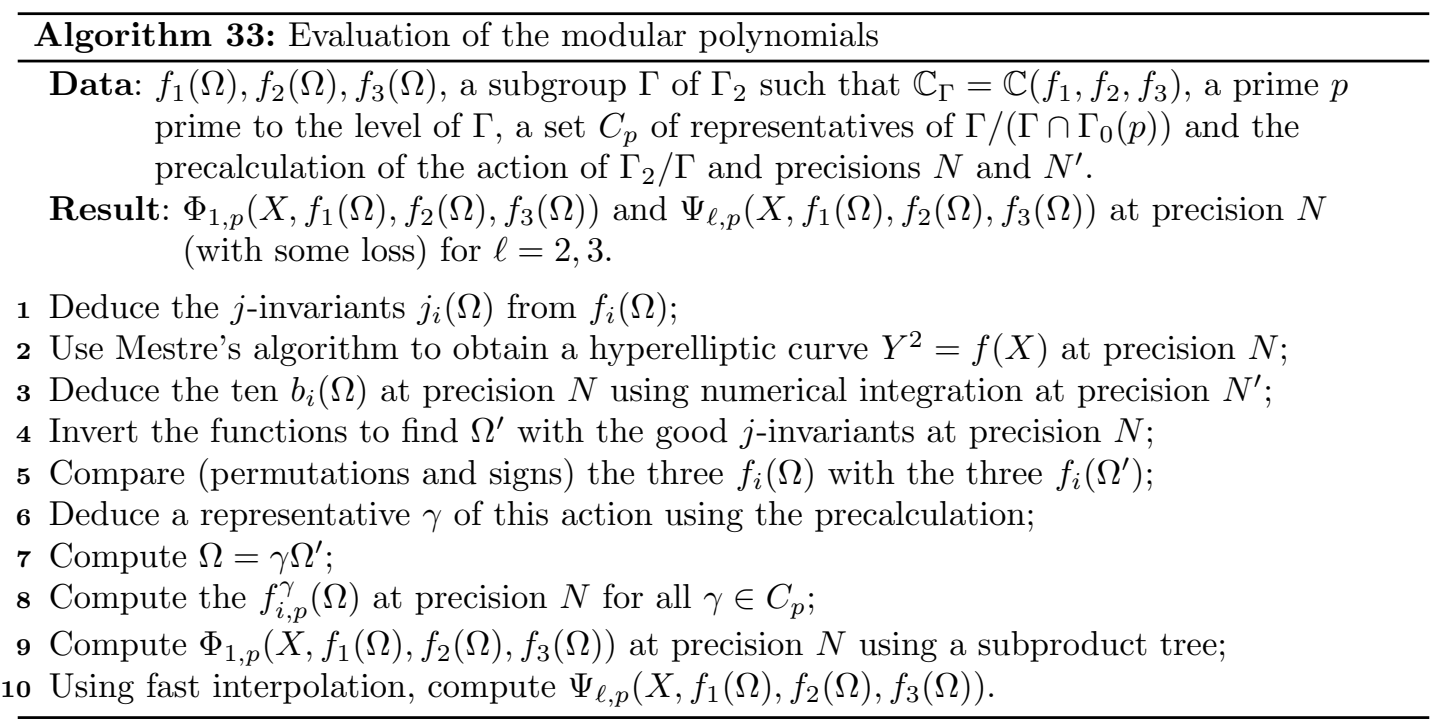

The complexity of the algorithm depends on the complexity of the evaluation of the $f_{i}$ at some $\Omega$. Let $q=p^{3}+p^{2}+p+1$. In the case of the theta constants and functions derived from them (as the $j$-invariants), Steps 1 to 7 are of complexity $O\left(\mathcal{M}^{\prime}(N) \log (N)\right)$ (by $\left[\mathbf{9}\right.$, Theorem 9.3]), Step 8 is of complexity $O\left(q \mathcal{M}^{\prime}(N) \log (N)\right)$ (by [16, Theorem 12] under Conjecture 23), Step $9 O(\mathcal{M}(q) \log (q))$ and Step $10 O(\mathcal{M}(q) \log (q))$, so that the complexity of this algorithm with functions derived from the theta constants is $O\left(q \mathcal{M}^{\prime}(N) \log (N)\right.$ $+\mathcal{M}(q) \log (q)) \subseteq \tilde{O}\left(p^{3} N\right)$. In practice, the limiting step is Step 8 (see $\S 6$ ).

Suppose $f_{1}$ is the variable which has the largest degree among all the numerators and denominators of the coefficients of the modular polynomials. Denote by $d_{T}^{A}$ (respectively $d_{T}^{B}$ ) the maximum of the total degrees of the numerators (respectively denominators) of the coefficients of the three modular polynomials and by $d_{f_{1}}$ (respectively $d_{f_{2}}, d_{f_{3}}$ ) the maximum exponent of the variable $f_{1}$ (respectively $f_{2}, f_{3}$ ) appearing in one of the coefficients of these three polynomials. Let $n=d_{T}^{A}+d_{T}^{B} \leqslant 6 d_{f_{1}}$. To obtain the modular polynomials, Algorithm 33 will be executed $(n+1)\left(d_{f_{2}}+1\right)\left(d_{f_{3}}+1\right)$ times and we will interpolate $3 q$ rational fractions. The complexity to compute the modular polynomials is then

$$
(n+1)\left(d_{f_{2}}+1\right)\left(d_{f_{3}}+1\right) \tilde{O}\left(p^{3} N\right)+\tilde{O}\left(n p^{3} d_{f_{2}} d_{f_{3}} N\right) \subseteq \tilde{O}\left(d_{f_{1}} d_{f_{2}} d_{f_{3}} p^{3} N\right)
$$

Note that we suppose that we know the degrees of all the trivariate rational fractions to use the Cauchy interpolation with the extended Euclidean algorithm. We discuss in $\S 6$ how to find these degrees.

As we do not have any explicit bounds on the size of the coefficients of the modular polynomials, we assume that it is sufficient to use a floating-point precision of $O(N)$ to do all the computations, where $N$ is the size of the largest coefficient, so that after the interpolation step it is possible to correctly round the coefficients. We also assume Assumption 24.

TheOREm 34 (Under Conjecture 23 and the heuristics of the preceding paragraph). Let $f_{1}, f_{2}, f_{3}$ be three modular functions derived from the theta constants for a congruence subgroup $\Gamma$ of $\Gamma_{2}$ generating the function field $\mathbb{C}_{\Gamma}$. Let $p$ be a prime number, prime to the level of $\Gamma$. Then, under the previous assumptions, the modular polynomials for these data can be 
computed in $\tilde{O}\left(d_{f_{1}} d_{f_{2}} d_{f_{3}} p^{3} N\right)$ time, if the degrees in the $f_{i}$ of all the coefficients (numerators and denominators) of the three modular polynomials are known and if $d_{f_{1}}=\max \left(d_{f_{1}}, d_{f_{2}}, d_{f_{3}}\right)$, where $N$ is the size of the largest coefficient of these modular polynomials.

\section{REMARK 35.}

- The conjecture is used for the complexity, but it does not affect the correctness of the algorithm because it is easy to check whether the period matrix found is the good one or not at each evaluation step.

- For the complexity $\tilde{O}\left(d_{f_{1}} d_{f_{2}} d_{f_{3}} p^{3} N\right)$ to be quasi-linear in the output size, we must assume that the average size of the coefficients of the modular polynomials is in $\Omega(N)$.

\section{Computational results}

Here, we present the modular polynomials we have computed with Streng invariants and with the $b_{i}^{\prime}$. The experimental findings given in this section and proved in the next one are used to optimize the implementation of the computation of the modular polynomials (see $\S 6$ ).

\subsection{Modular polynomials with the invariants of Streng}

With the algorithm we have presented in $\S 3.1$, Régis Dupont [9] has calculated the modular polynomials with Igusa invariants for $p=2$, but because of the large size of the coefficients and the large degrees of the rational fractions in $j_{1}, j_{2}$ and $j_{3}$, he has calculated only the denominators for $p=3$ and the degrees of the rational fractions.

We begin with some notation to compare the results found with the Igusa and the Streng invariants (see Definitions 13 and 28). For $p=2$, the number of isogenies is $p^{3}+p^{2}+p+1=15$. Denote for $\ell=2,3$

$$
\Phi_{1,2}(X)=X^{15}+\sum_{i=0}^{14} \frac{A_{1, i}\left(i_{1}, i_{2}, i_{3}\right)}{B_{1, i}\left(i_{1}, i_{2}, i_{3}\right)} X^{i} \quad \text { and } \quad \Psi_{\ell, 2}(X)=\sum_{i=0}^{14} \frac{A_{\ell, i}\left(i_{1}, i_{2}, i_{3}\right)}{B_{\ell, i}\left(i_{1}, i_{2}, i_{3}\right)} X^{i} .
$$

We consider the quotient $A_{j, i} / B_{j, i}$ as the $i$ th coefficient of the $j$ th modular polynomial. The numerator and the denominator of each coefficient are polynomials in $\mathbb{Z}\left[i_{1}, i_{2}, i_{3}\right]$.

We recall that Dupont found that the denominators of the three polynomials were of the form $1428 j_{1}^{\alpha} D_{2}\left(j_{1}, j_{2}, j_{3}\right)^{6}$ for some integer $\alpha$ ranging between 5 and 21 and $D_{2}$ of degrees 5,7 and 5 in $j_{1}, j_{2}$, and $j_{3}$ respectively (see $\left[\mathbf{9}\right.$, pp. 225-226] for these results and for the definition of $D_{2}$ ). With Streng invariants, we have found that the denominators are of the form $c i_{3}^{\alpha} D_{2}^{\prime}\left(i_{1}, i_{2}, i_{3}\right)$ for $\Phi_{1,2}$ and of the form $c i_{3}^{\alpha}\left(D_{2}^{\prime}\left(i_{1}, i_{2}, i_{3}\right)\right)^{2}$ for the others, where $c$ is a constant in $\mathbb{Z}, \alpha$ varies from 0 to 3 , and $D_{2}^{\prime}=\left(24576 i_{3} i_{1}^{5}+\left(96 i_{2}^{3}-4608 i_{3} i_{2}\right) i_{1}^{4}+\left(-6220800 i_{3} i_{2}-12288 i_{3}^{2}\right) i_{1}^{3}+\right.$ $\left(-23328 i_{2}^{4}-48 i_{3} i_{2}^{3}+1088640 i_{3} i_{2}^{2}+2304 i_{3}^{2} i_{2}+24883200 i_{3}^{2}\right) i_{1}^{2}+\left(93312 i_{3} i_{2}^{3}+419904000 i_{3} i_{2}^{2}-\right.$ $\left.5909760 i_{3}^{2} i_{2}+\left(1536 i_{3}^{3}-8398080000 i_{3}^{2}\right)\right) i_{1}+\left(1417176 i_{2}^{5}-5832 i_{3} i_{2}^{4}+\left(6 i_{3}^{2}-94478400 i_{3}\right) i_{2}^{3}+\right.$ $\left.\left.287712 i_{3}^{2} i_{2}^{2}+\left(-288 i_{3}^{3}+1154736000 i_{3}^{2}\right) i_{2}+\left(-248832 i_{3}^{3}+755827200000 i_{3}^{2}\right)\right)\right)$ is irreducible. It is clear that the exponents of $D_{2}$ and $D_{2}^{\prime}$ are related to the exponent of $h_{10}$ in the definition of the different $j$-invariants. Denote the degree of the numerator of the $\ell$ th coefficient of the $i$ th modular polynomial in $i_{j}$ by $d_{i, j, \ell}$ (see Definition 27) and the exponent of $j_{3}$ appearing in the denominator of the $\ell$ th coefficient of the $i$ th polynomial by $\alpha_{i, \ell}$. The degrees found are written in Table 1.

The degrees of the numerators of the coefficients of the modular polynomials found by Dupont for the Igusa invariants vary from 37 to 60 in $j_{1}$, from 50 to 75 in $j_{2}$ and from 33 to 50 in $j_{3}$ for $\Phi_{1,2}(X)$ while they do not exceed 25 with Streng invariants. The size of the integers in the former case is bounded by 210 decimal digits and by 105 in the latter case. Moreover, 
the three polynomials computed by Dupont (and accessible at his website) fill $57 \mathrm{MB}$ and the others 2.1 MB. Thus, the Streng invariants provide smaller modular polynomials in terms of degree, precision and total space.

We also managed to compute the three modular polynomials with Streng invariants for $p=3$. The number of isogenies is 40 . The denominators have the same properties as described before: they are of the form $c i_{3}^{\alpha}\left(D_{3}^{\prime}\left(i_{1}, i_{2}, i_{3}\right)\right)^{2}$ for $\Phi_{1,3}$ and of the form $c i_{3}^{\alpha}\left(D_{3}^{\prime}\left(i_{1}, i_{2}, i_{3}\right)\right)^{4}$ for the others. The common part $D_{3}^{\prime}$ is an irreducible polynomial which occurs with degrees 13 , 10 and 8 in $i_{1}, i_{2}$ and $i_{3}$ respectively. Dupont has found that the denominators with the Igusa invariants are of the form $c j_{1}^{\alpha} D_{3}\left(j_{1}, j_{2}, j_{3}\right)^{18}$, where $D_{3}$ has degrees 14,20 and 13 in $j_{1}, j_{2}$ and $j_{3}$ respectively. We present some degrees of the numerators in Table 2.

The degrees are far smaller than those with the Igusa invariants which range from 243 to 420. We do not know the size of the integers of the polynomial with Igusa invariants, but in the case of Streng invariants we have found that they can reach 550 decimal digits. The three polynomials fill $890 \mathrm{MB}$.

\subsection{Modular polynomials with the $b_{i}^{\prime}$}

We have computed the modular polynomials for $b_{i}^{\prime}$ for $p=3,5$ and 7 (see (6) for their definition). Note that for $p=2$, these polynomials do not exist because $\Gamma(2,4) \cap \Gamma_{0}(2)=$ $\Gamma(2,4)$. This time, there is only one common denominator $D_{p}$ for all the coefficients of the

TABLE 1. Degrees of the numerators of the modular polynomials with Streng invariants for $p=2$.

\begin{tabular}{c|cccc|cccc|cccc}
\hline$\ell$ & $d_{1,1, \ell}$ & $d_{1,2, \ell}$ & $d_{1,3, \ell}$ & $\alpha_{1, \ell}$ & $d_{2,1, \ell}$ & $d_{2,2, \ell}$ & $d_{2,3, \ell}$ & $\alpha_{2, \ell}$ & $d_{3,1, \ell}$ & $d_{3,2, \ell}$ & $d_{3,3, \ell}$ & $\alpha_{3, \ell}$ \\
\hline 0 & 25 & 11 & 11 & 3 & 30 & 17 & 15 & 3 & 33 & 17 & 16 & 3 \\
1 & 23 & 11 & 11 & 3 & 28 & 17 & 15 & 3 & 31 & 17 & 16 & 3 \\
2 & 23 & 11 & 11 & 3 & 28 & 17 & 15 & 3 & 31 & 17 & 16 & 3 \\
3 & 21 & 11 & 11 & 3 & 26 & 17 & 15 & 3 & 29 & 17 & 16 & 3 \\
4 & 21 & 11 & 11 & 3 & 26 & 17 & 15 & 3 & 29 & 17 & 16 & 3 \\
5 & 20 & 11 & 10 & 3 & 25 & 17 & 14 & 3 & 28 & 17 & 15 & 3 \\
6 & 20 & 11 & 10 & 3 & 25 & 17 & 14 & 3 & 28 & 17 & 15 & 3 \\
7 & 18 & 10 & 9 & 2 & 23 & 17 & 14 & 3 & 26 & 17 & 15 & 3 \\
8 & 18 & 10 & 9 & 2 & 23 & 16 & 13 & 2 & 26 & 16 & 14 & 2 \\
9 & 16 & 10 & 8 & 2 & 21 & 15 & 12 & 2 & 24 & 15 & 13 & 2 \\
10 & 16 & 8 & 7 & 1 & 21 & 15 & 12 & 2 & 24 & 15 & 13 & 2 \\
11 & 15 & 8 & 7 & 1 & 20 & 13 & 11 & 1 & 23 & 13 & 12 & 1 \\
12 & 15 & 7 & 7 & 1 & 20 & 13 & 11 & 1 & 23 & 13 & 12 & 1 \\
13 & 11 & 6 & 5 & 0 & 16 & 12 & 10 & 1 & 20 & 12 & 11 & 1 \\
14 & 8 & 5 & 4 & 0 & 13 & 11 & 8 & 0 & 16 & 11 & 9 & 0 \\
\hline
\end{tabular}

TABLE 2. Degrees of the numerators of the modular polynomials with Streng invariants for $p=3$.

\begin{tabular}{c|cccc|cccc|cccc}
\hline$\ell$ & $d_{1,1, \ell}$ & $d_{1,2, \ell}$ & $d_{1,3, \ell}$ & $\alpha_{1, \ell}$ & $d_{2,1, \ell}$ & $d_{2,2, \ell}$ & $d_{2,3, \ell}$ & $\alpha_{2, \ell}$ & $d_{3,1, \ell}$ & $d_{3,2, \ell}$ & $d_{3,3, \ell}$ & $\alpha_{3, \ell}$ \\
\hline 0 & 61 & 32 & 32 & 4 & 87 & 52 & 48 & 4 & 92 & 52 & 49 & 4 \\
1 & 61 & 32 & 31 & 4 & 87 & 52 & 47 & 4 & 92 & 52 & 48 & 4 \\
2 & 61 & 32 & 31 & 4 & 87 & 52 & 47 & 4 & 92 & 52 & 48 & 4 \\
$\vdots$ & & $\vdots$ & $\vdots$ & & & $\vdots$ & $\vdots$ & & & $\vdots$ & $\vdots$ & \\
37 & 41 & 22 & 21 & 1 & 67 & 43 & 37 & 1 & 72 & 43 & 39 & 1 \\
38 & 36 & 21 & 19 & 0 & 62 & 42 & 36 & 1 & 67 & 42 & 37 & 1 \\
39 & 31 & 20 & 17 & 0 & 57 & 41 & 33 & 0 & 62 & 41 & 35 & 0 \\
\hline
\end{tabular}


three polynomials (there are no constants and no powers of one of the $b_{i}^{\prime}$ ). For example, we have $D_{3}=1024 b_{3}^{\prime 6} b_{2}^{\prime 6} b_{1}^{\prime 10}-\left(\left(768 b_{3}^{\prime 8}+1536 b_{3}^{\prime 4}-256\right) b_{2}^{\prime 8}+1536 b_{3}^{\prime 8} b_{2}^{\prime 4}-256 b_{3}^{\prime 8}\right) b_{1}^{\prime 8}+\left(1024 b_{3}^{\prime 6} b_{2}^{\prime 10}+\right.$ $\left.\left(1024 b_{3}^{\prime 10}+2560 b_{3}^{\prime 6}-512 b_{3}^{\prime 2}\right) b_{2}^{\prime 6}-\left(512 b_{3}^{\prime 6}-64 b_{3}^{\prime 2}\right) b_{2}^{\prime 2}\right) b_{1}^{\prime 6}-\left(1536 b_{3}^{\prime 8} b_{2}^{\prime 8}+\left(-416 b_{3}^{\prime 4}+32\right) b_{2}^{\prime 4}+\right.$ $\left.32 b_{3}^{\prime 4}\right) b_{1}^{\prime 4}-\left(\left(512 b_{3}^{\prime 6}-64 b_{3}^{\prime 2}\right) b_{2}^{\prime 6}-64 b_{3}^{\prime 6} b_{2}^{\prime 2}\right) b_{1}^{\prime 2}+256 b_{3}^{\prime 8} b_{2}^{\prime 8}-32 b_{3}^{\prime 4} b_{2}^{\prime 4}+1$.

For $p=5$ (respectively $p=7$ ), the denominator occurs with exponents 70 (respectively 226) in the three $b_{i}^{\prime}$. These three denominators have interesting properties. They are symmetric, the exponents of the $b_{i}^{\prime}$ are always even and there are relations modulo 2 and 4 between the exponents of each monomial. We have also noted similar properties for the numerators. In particular, we have noted that for $p=3$ and $5, \Psi_{2, p}\left(X, b_{1}^{\prime}, b_{2}^{\prime}, b_{3}^{\prime}\right)=\Psi_{3, p}\left(X, b_{1}^{\prime}, b_{3}^{\prime}, b_{2}^{\prime}\right)$ and $\Phi_{1, p}\left(X, b_{1}^{\prime}, b_{2}^{\prime}, b_{3}^{\prime}\right)=\Phi_{1, p}\left(X, b_{1}^{\prime}, b_{3}^{\prime}, b_{2}^{\prime}\right)$. Moreover, the total degrees for the denominators are 24, 120 and 226, which always seems to be $p^{3}-p$. We will prove all this in the next section.

Table 3 shows a few of the degrees for $p=3$. This table can be compared with the results found with the $j$-invariants (see Table 2). The notation is similar to before.

TABle 3. Degrees of the numerators of the modular polynomials with the $b_{i}^{\prime}$ for $p=3$.

\begin{tabular}{c|ccc|ccc}
\hline$\ell$ & $d_{1,1, \ell}$ & $d_{1,2, \ell}$ & $d_{1,3, \ell}$ & $d_{2,1, \ell}$ & $d_{2,2, \ell}$ & $d_{2,3, \ell}$ \\
\hline 0 & 40 & 10 & 10 & 37 & 13 & 12 \\
1 & 37 & 12 & 12 & 36 & 15 & 14 \\
2 & 38 & 14 & 14 & 37 & 17 & 16 \\
3 & 39 & 16 & 16 & 36 & 19 & 18 \\
4 & 36 & 16 & 16 & 35 & 19 & 18 \\
$\vdots$ & & $\vdots$ & & & $\vdots$ & \\
35 & 21 & 16 & 16 & 22 & 19 & 18 \\
36 & 20 & 16 & 16 & 19 & 19 & 18 \\
37 & 17 & 16 & 16 & 16 & 17 & 16 \\
38 & 14 & 14 & 14 & 15 & 15 & 14 \\
39 & 13 & 12 & 12 & 12 & 13 & 12 \\
\hline
\end{tabular}

Table 4 indicates the minimal and maximal degrees each of the $b_{i}^{\prime}$ take for the different modular polynomials for $p=5$ and 7 .

TABLE 4. Degrees of the numerators of the modular polynomials with the $b_{i}^{\prime}$ for $p=5,7$.

\begin{tabular}{c|c|c|c}
\hline $\min -\max$ of & $b_{1}^{\prime}$ & $b_{2}^{\prime}$ & $b_{3}^{\prime}$ \\
\hline$\Phi_{1,5}$ & $75-156$ & $70-92$ & $70-92$ \\
$\Psi_{2,5}$ & $72-155$ & $75-97$ & $72-94$ \\
$\Phi_{1,7}$ & $233-400$ & $226-272$ & $226-272$ \\
$\Psi_{2,7}$ & $230-397$ & $233-279$ & $230-276$ \\
\hline
\end{tabular}

The integers have about 10,60 and 190 decimal digits for $p=3,5$ and 7 respectively. The three polynomials fill $270 \mathrm{~KB}$ for $p=3$ (which is 3000 times smaller than the total space of the modular polynomials with Streng invariants for $p=3$ ), and $305 \mathrm{MB}$ for $p=5$, while only the first two fill 29 GB for $p=7$ (we have not computed the third because it would have taken too much time and we have assumed that there is the same symmetry as in the cases $p=3$ and $p=5$, so that the third polynomial can be deduced from the second one). Compared with the polynomials found with the Streng invariants for $p=3$, these invariants produce smaller polynomials in terms of degree, precision and total space. 


\section{Analysis of the results}

\subsection{Humbert surfaces}

In this section we will examine the meaning of the denominators appearing in the different modular polynomials. The principal tool we use is the notion of Humbert surfaces, which have been studied in $[\mathbf{2 1}]$.

Let $\Delta \equiv 0,1 \bmod 4$ and $\Delta>0$. We call the Humbert surface $H_{\Delta}$ of discriminant $\Delta$ the irreducible surface of matrices which are equivalent to some $\Omega=\left(\begin{array}{l}\Omega_{1} \\ \Omega_{2} \Omega_{2}\end{array}\right)$ in $\Gamma_{2} \backslash \mathcal{H}_{2}$ satisfying $k \Omega_{1}+\ell \Omega_{2}-\Omega_{3}=0$, where $k$ and $\ell$ are determined uniquely by $\Delta=4 k+\ell$ and $\ell \in\{0,1\}$.

These surfaces are of particular interest to us because of the next proposition which states that $\mathcal{L}_{p}=H_{p^{2}}$.

Proposition 36. Let $m$ be a positive integer. Then the Humbert surface $H_{m^{2}}$ is the moduli space for isomorphism classes of principally polarized abelian surfaces that split as a product of two elliptic curves via an isogeny of degree $m^{2}$.

Proof. See [21, Proposition 2.14].

For each discriminant $\Delta$ there is an irreducible polynomial $L_{\Delta}\left(j_{1}, j_{2}, j_{3}\right)$ whose zero set is the Humbert surface of discriminant $\Delta$. Thus, by Lemma $17, L_{p^{2}}\left(j_{1}, j_{2}, j_{3}\right)$ divides the denominators of the modular polynomials with the Igusa invariants. The exponent to which $L_{p^{2}}\left(j_{1}, j_{2}, j_{3}\right)$ appears in the denominator seems to depend on the exponent of the $h_{10}$ in the definition of the $j$-invariants. A heuristic reason for the factor $j_{1}^{\alpha}$ in the denominator of a coefficient of a modular polynomial is to compensate for the case where $h_{12}(\Omega)=0$ (recall Definition 13). With Streng invariants, there is a factor $i_{3}^{\alpha}$ to compensate for the case where $h_{4}(\Omega)=0$ (recall Definition 28). Note that $j_{1}$ (respectively $i_{3}$ ) has the greatest exponent of $h_{12}$ (respectively $h_{4}$ ) in its definition among $j_{1}, j_{2}, j_{3}$ (respectively $i_{1}, i_{2}, i_{3}$ ).

Moreover, a formula for the degree of these surfaces exists. Let

$$
a_{p^{2}}:=24 \sum_{\substack{x \in \mathbb{Z}, 4 \mid\left(p^{2}-x^{2}\right)}} \sigma_{1}\left(\frac{p^{2}-x^{2}}{4}\right)+12 p^{2}-2
$$

with $\sigma_{1}(n)=\sum_{d \mid n} d$ the sum of positive divisors function. Then we have the following theorem.

Theorem 37. The degree of any Humbert surface of discriminant $p^{2}$ can be obtained by the formula

$$
v\left(p^{2}\right) \operatorname{deg}\left(H_{p^{2}}\right)+5=\frac{a_{p^{2}}}{2} \quad \text { where } v\left(p^{2}\right)= \begin{cases}1 / 2 & \text { if } p=2, \\ 1 & \text { otherwise. }\end{cases}
$$

Proof. See [21, Theorem 3.8].

Applying this formula gives $\operatorname{deg}\left(H_{4}\right)=60$ and $\operatorname{deg}\left(H_{9}\right)=120$. Here, the degree of the surfaces is the degree of the homogenous form of $L_{\Delta}$ with weight $(4,6,10,12)$ for the functions $\left(h_{4}, h_{6}, h_{10}, h_{12}\right)$ (see [23, pp. 170-172]). We have then substituted the $j$-invariants of the common denominator for $p=2$ and $p=3$ by their definition in terms of the $h_{i}$, and multiplied by a power of $h_{10}$ to homogenize. The degree we have found for $p=2$ is 100 (respectively 300) with the Streng invariants (respectively Igusa), but there is a factor $h_{4}^{10}$ (respectively $h_{12}^{20}$ ) and we have $100-40=60$ (respectively $300-240=60$ ). This factor can be explained by the fact that the $j$-invariants are zero when $h_{4}=0$ (respectively $h_{12}=0$ ). For $p=3$, we have found (for Streng invariants) that the degree is 200 and there is a factor $h_{4}^{20}$. We have then $200-80=120$. 
We now study what happens to our modular polynomials with the theta constants. We have another formula for the degree, due to the work of Runge ([37], see also [21]) who considered finite covers of $\Gamma_{2} \backslash \mathcal{H}_{2}$ for the study of Humbert surfaces because of the large degrees and coefficients of the polynomial with the $j$-invariants. Define $\Gamma^{*}(2,4)$ to be the largest normal subgroup of $\Gamma(2,4)$ which does not contain the matrix $\operatorname{diag}(-1,1,-1,1)$. The natural projection $\pi: \Gamma^{*}(2,4) \backslash \mathcal{H}_{2} \rightarrow \Gamma_{2} \backslash \mathcal{H}_{2}$ is a finite map. We say that each component of $\pi^{-1}\left(H_{p^{2}}\right)$ in $\Gamma^{*}(2,4) \backslash \mathcal{H}_{2}$ is a Humbert component and it is possible to define an order $v_{i}^{\prime}\left(p^{2}\right)$ for each irreducible Humbert component $F_{p^{2}, i}$. Since $\Gamma^{*}(2,4)$ is normal, these components have the same degree. Moreover by [37], any irreducible component of the covering of $H_{p^{2}}$ is given by the zero set of a single irreducible polynomial.

Proposition 38. The degree of any Humbert component $F_{p^{2}, i}$ in $\Gamma_{2,4}^{*} \backslash \mathcal{H}_{2}$ is given by the formula

$$
a_{p^{2}}=10\left(1+\operatorname{deg}\left(F_{p^{2}, i}\right)\right)
$$

Proof. See [21, Proposition 3.9].

Proposition 39. Let $p>2$ be a prime number. The degree of $F_{p^{2}, i}$ is $p^{3}-p$.

Proof. From the degree formula above, we have that $a_{p^{2}}=10\left(1+\operatorname{deg}\left(F_{p^{2}, i}\right)\right)$ and from the definition of $a_{p^{2}}$ it suffices to prove that $\sum_{x>0} \sigma_{1}\left(\left(p^{2}-x^{2}\right) / 4\right)=\left(5 p^{3}-6 p^{2}-5 p+6\right) / 24$. The left-hand side can be rewritten as $\frac{1}{2} \sum_{k=1}^{p} \sigma_{1}(k) \sigma_{1}(p-k)$ and the result comes from the equality $(1 / 2 i \pi) G_{2}^{\prime}(\Omega)=\frac{5}{6} G_{4}(\Omega)-2 G_{2}(\Omega)^{2}$ of $[\mathbf{4 6}]$, where $G_{i}$ is the $i$ th Eisenstein series (in genus 1). (Moreover, using the fact that $\sigma_{1}$ is multiplicative, it can be shown that for all $p>2$, the degree of $F_{4 p^{2}, i}$ is also $p^{3}-p$, but we do not need this result.)

In our case, we use $\Gamma(2,4)$ and not $\Gamma^{*}(2,4)$, but we noted that the total degree found for $p=3,5$ and 7 is always $p^{3}-p$. The reason for this is that the degree formula depends on the number of Humbert components and the order of some isotropy subgroup and these numbers are equal for the groups $\Gamma^{*}(2,4)$ and $\Gamma(2)$ (see [21]) so that it is the case for $\Gamma(2,4)$ because $\Gamma^{*}(2,4)<\Gamma(2,4)<\Gamma(2)$. Thus, the degree formula of a component of discriminant $p^{2}$ for the group $\Gamma(2,4)$ is the same as those for the group $\Gamma^{*}(2,4)$, namely $p^{3}-p$. Note that the definition of degree here is the total degree of the polynomial because the $\theta_{i}(\tau / 2)$ are Siegel modular forms of weight 1 for $\Gamma(2,4)$, up to a root of unity.

Consider this time the locus $\mathcal{L}_{p}^{\prime}$ of all the principally polarized abelian surfaces modulo $\Gamma(2,4)$ that are $(p, p)$-isogenous to a principally polarized abelian surface $\Omega$ which is isogenous to a product of two elliptic curves by the $(2,2)$-isogeny $\Omega \rightarrow \Omega / 2$ and such that $\theta_{0}(\Omega / 2)=0$ (recall that $b_{i}^{\prime}(\Omega):=\theta_{i}(\Omega / 2) / \theta_{0}(\Omega / 2)$ and Proposition 12$)$.

Proposition 40. The denominators of the modular polynomials for the functions $b_{1}^{\prime}, b_{2}^{\prime}, b_{3}^{\prime}$ are divisible by a polynomial $L_{p}^{\prime}$ in $\mathbb{Q}\left[b_{1}^{\prime}, b_{2}^{\prime}, b_{3}^{\prime}\right]$ describing the preceding locus.

Proof. We adapt the proof of lemma 6.2 of [5]. Let $\Omega \in \Gamma(2,4) \backslash \mathcal{H}_{2}$ which is $(p, p)$-isogenous to $\Omega^{\prime}$ such that $\theta_{0}\left(\Omega^{\prime} / 2\right)=0$. Let $c$ be a coefficient of the polynomial $\Phi_{1, p}$. For some $\gamma \in \Gamma(2,4) /\left(\Gamma(2,4) \cap \Gamma_{0}(p)\right), b_{1, p}^{\prime}(\gamma \Omega)$ is infinite. The evaluation of $c$ at $\Omega$ is a symmetric expression in the $b_{1, p}^{\prime \gamma}(\Omega)$ s. Generically, there is no algebraic relationship between these values, and the evaluation of $c$ at $\Omega$ is, therefore, infinite. Since the $b_{i}^{\prime}(\Omega)$ are finite, the numerator of $c$ is finite. We conclude that the denominator of $c$ must vanish at $\Omega$, which means that $c$ is divisible by a polynomial describing the locus. The proof for $\Phi_{\ell, p}, \ell=2,3$ proceeds similarly. 
We have noticed that for $p=3,5$ and 7 , the coefficients of the three modular polynomials with the $b_{i}^{\prime}$ always have $L_{p}^{\prime}$ has denominator (unlike the case with the $j$-invariants where there also is a factor $j_{1}$ or $i_{3}$, as explained in $\S 4$ ). This justifies the following conjecture, which will be used in the next sections.

ConjeCture 41. The polynomial $L_{p}^{\prime}$ is the denominator of all the coefficients of the three modular polynomials (except for the coefficient of degree $p^{3}+p^{2}+p+1$ of $\Phi_{1, p}(X)$ which is equal to 1$)$.

\subsection{Symmetries}

As mentioned above $(\S 4.2)$, we have noticed for $p=3,5$ that $\Psi_{2, p}\left(X, b_{1}^{\prime}, b_{2}^{\prime}, b_{3}^{\prime}\right)=\Psi_{3, p}\left(X, b_{1}^{\prime}\right.$, $\left.b_{3}^{\prime}, b_{2}^{\prime}\right)$ and for $p=3,5,7$ that $\Phi_{1, p}\left(X, b_{1}^{\prime}, b_{2}^{\prime}, b_{3}^{\prime}\right)=\Phi_{1, p}\left(X, b_{1}^{\prime}, b_{3}^{\prime}, b_{2}^{\prime}\right)$. These symmetries have the following meaning. For each variety $\Omega \in \Gamma(2,4)$ and $p \Omega$ having invariants $\left(b_{1}^{\prime}(\Omega), b_{2}^{\prime}(\Omega)\right.$, $\left.b_{3}^{\prime}(\Omega)\right)$ and $\left(b_{1, p}^{\prime}(\Omega), b_{2, p}^{\prime}(\Omega), b_{3, p}^{\prime}(\Omega)\right)$, there exists a variety with invariants $\left(b_{1}^{\prime}(\Omega), b_{3}^{\prime}(\Omega), b_{2}^{\prime}(\Omega)\right)$ such that one of its $(p, p)$-isogenous varieties has invariants $\left(b_{1}^{\prime}(p \Omega), b_{3}^{\prime}(p \Omega), b_{2}^{\prime}(p \Omega)\right)$.

A proof of this can be obtained by looking at the action of some matrices. Indeed, we have that $\Phi_{1, p}$ is the minimal polynomial of $b_{1, p}$, which means it is the unique irreducible monic polynomial such that for all $\Omega \in \mathcal{H}_{2}, \Phi_{1, p}\left(x, b_{1}^{\prime}(\Omega), b_{2}^{\prime}(\Omega), b_{3}^{\prime}(\Omega)\right)=0$ if and only if $x=b_{1, p}^{\prime}(\gamma \Omega)$ for some $\gamma \in \Gamma_{2}$; hence $\Phi_{1, p}\left(b_{1, p}^{\prime}(\gamma \Omega), b_{1}^{\prime}(\gamma \Omega), b_{2}^{\prime}(\gamma \Omega), b_{3}^{\prime}(\gamma \Omega)\right)=0$ for all $\gamma \in \Gamma_{2}$. What we are looking for is a matrix that fixes $b_{1}^{\prime}$ and $b_{1, p}^{\prime}$ and interchanges $b_{2}^{\prime}$ with $b_{3}^{\prime}$ and $b_{2, p}^{\prime}$ with $b_{3, p}^{\prime}$. This action on $\Phi_{1, p}(X)$ would provide a unitary polynomial with the same roots and degree as $\Phi_{1, p}(X)$ and since $\Phi_{1, p}(X)$ is a minimal polynomial, they have to be equal.

Assume that we have the symmetry for $\Phi_{1, p}$. Then, by Definition 27 , we have $b_{\ell, p}^{\prime}=$ $\Psi_{\ell, p}\left(b_{1, p}^{\prime}\right) / \Phi_{1, p}^{\prime}\left(b_{1, p}^{\prime}\right)$ for $\ell=2,3$. We use this action on $\Psi_{2, p}(X)$ which gives us

$$
b_{3, p}^{\prime}=\Psi_{2, p}\left(b_{1, p}^{\prime}, b_{1}^{\prime}, b_{3}^{\prime}, b_{2}^{\prime}\right) / \Phi_{1, p}^{\prime}\left(b_{1, p}^{\prime}, b_{1}^{\prime}, b_{2}^{\prime}, b_{3}^{\prime}\right),
$$

so that

$$
\Psi_{2, p}\left(b_{1, p}^{\prime}, b_{1}^{\prime}, b_{3}^{\prime}, b_{2}^{\prime}\right)=b_{3, p}^{\prime} \Phi_{1, p}^{\prime}\left(b_{1, p}^{\prime}, b_{1}^{\prime}, b_{2}^{\prime}, b_{3}^{\prime}\right)=\Psi_{3, p}\left(b_{1, p}^{\prime}, b_{1}^{\prime}, b_{2}^{\prime}, b_{3}^{\prime}\right) .
$$

Firstly, the search is done among the representatives of $\Gamma_{2} / \Gamma(2,4)$ because $\Gamma(2,4)$ fixes the $b_{i}^{\prime}$. A representative of the unique class such that $\left(b_{1}^{\prime \gamma}, b_{2}^{\prime \gamma}, b_{3}^{\prime \gamma}\right)=\left(b_{1}^{\prime}, b_{3}^{\prime}, b_{2}^{\prime}\right)$ is

$$
\gamma=\left(\begin{array}{cccc}
1 & -3 & -2 & 2 \\
0 & 1 & 2 & 0 \\
0 & 0 & 1 & 0 \\
0 & -4 & -5 & 1
\end{array}\right)
$$

Secondly, we look for a matrix $\gamma^{\prime}$ in $\Gamma(2,4)$ such that $\gamma \gamma^{\prime} \in \Gamma_{0}(p)$. For $p=3,5$ and 7 we can take for $\gamma^{\prime}$ respectively

$$
\left(\begin{array}{cccc}
-5 & 24 & -12 & 12 \\
-2 & 19 & -12 & 8 \\
0 & 6 & -5 & 2 \\
-2 & 4 & 0 & 3
\end{array}\right), \quad\left(\begin{array}{cccc}
-7 & 6 & 4 & 2 \\
0 & -7 & 2 & 0 \\
0 & 10 & -3 & 0 \\
10 & -8 & -6 & -3
\end{array}\right) \quad \text { and } \quad\left(\begin{array}{cccc}
13 & 12 & -16 & -6 \\
-10 & -3 & 10 & 4 \\
56 & 14 & -55 & -22 \\
30 & -40 & -12 & -7
\end{array}\right)
$$

Recall that for a matrix $X$, we denote by $X_{0}$ the vector composed of the diagonal entries of $X$.

Lemma 42. Let $M=\left(\begin{array}{cc}A^{\prime} & B^{\prime} \\ C^{\prime} & D^{\prime}\end{array}\right) \in \Gamma_{2} / \Gamma(2,4)$ and $M^{\prime} \in \Gamma(2,4)$ such that $M M^{\prime} \in \Gamma_{0}(p)$, for some prime $p>2$. Then $\left(M M^{\prime}\right)_{p}$ is in the same equivalence class as $M$ for all $p \equiv 1 \bmod 4$. For $p \equiv 3 \bmod 4$, this is the case if we have the additional properties $\left(A^{\prime t} B^{\prime}\right)_{0} \equiv 0 \bmod 2$ and $\left(C^{\prime} D^{\prime}\right)_{0} \equiv 0 \bmod 2$. 
Proof. Let $M M^{\prime}=\left(\begin{array}{cc}A & B \\ C\end{array}\right)$. We study the conditions when $\left(M M^{\prime}\right)_{p} M^{-1} \in \Gamma(2,4)$ or, equivalently, when $\left(M M^{\prime}\right)_{p}\left(M M^{\prime}\right)^{-1}$ is in $\Gamma(2,4)$. We have

$$
\left(\begin{array}{cc}
A & p B \\
C / p & D
\end{array}\right)\left(\begin{array}{cc}
{ }^{t} D & -{ }^{t} B \\
-{ }^{t} C & { }^{t} A
\end{array}\right)=\left(\begin{array}{cc}
A^{t} D-p B^{t} C & -A^{t} B+p B^{t} A \\
C / p^{t} D-D^{t} C & -C / p^{t} B+D^{t} A
\end{array}\right)
$$

where $\left(\begin{array}{cc}{ }^{t} D & -{ }^{t} B \\ -{ }^{t} C & { }^{t} A\end{array}\right)$ is the inverse of $M$ by equation (1). As $p \equiv 1 \bmod 2$, this product is the identity modulo 2. Now for $p \equiv 1 \bmod 4$, we have $-A^{t} B+p B^{t} A \equiv C / p^{t} D-D^{t} C \equiv 0 \bmod 4$ (recall that this product is in $\left.\Gamma_{2}\right)$ so that $\left(M M^{\prime}\right)_{p}\left(M M^{\prime}\right)^{-1} \in \Gamma(2,4)$. For $p \equiv 3 \bmod 4$, we have $-A^{t} B+p B^{t} A \equiv 2 A^{t} B \bmod 4$ and $C / p^{t} D-D^{t} C \equiv 2 C^{t} D \bmod 4$. Thus, to be in $\Gamma(2,4)$, we want $\left(A^{t} B\right)_{0} \equiv\left(C^{t} D\right)_{0} \equiv 0 \bmod 2$. Finally, note that $M^{\prime} \equiv I_{4} \bmod 2$ and we deduce the lemma.

By this lemma, we have that $\left(\gamma \gamma^{\prime}\right)_{p}$ is in the same equivalence class as $\gamma$ for any prime $p>2$ and hence the permutation $\left(b_{1, p}^{\prime \gamma \gamma^{\prime}}, b_{2, p}^{\prime \gamma \gamma^{\prime}}, b_{3, p}^{\prime \gamma \gamma^{\prime}}\right)=\left(b_{1, p}^{\prime}, b_{3, p}^{\prime}, b_{2, p}^{\prime}\right)$. Moreover, the surjectivity of $\mathrm{Sp}_{4}(\mathbb{Z}) \rightarrow \mathrm{Sp}_{4}(\mathbb{Z} / 4 p \mathbb{Z})$ and the Chinese remainder theorem prove that the matrix $\gamma^{\prime}$ always exists. Thus, there are these symmetries for all prime $p>2$ (see Theorem 44).

By the above, we have also proved that the denominator is always symmetric in $b_{2}^{\prime}$ and $b_{3}^{\prime}$. To prove that $L_{p}^{\prime}$ is also symmetric in $b_{1}^{\prime}$ and $b_{2}^{\prime}$ (respectively $b_{1}^{\prime}$ and $b_{3}^{\prime}$ ), we use the matrices

$$
\gamma_{410}=\left(\begin{array}{cccc}
0 & -1 & 0 & 0 \\
-1 & 0 & 0 & 0 \\
0 & 0 & 0 & -1 \\
0 & 0 & -1 & 0
\end{array}\right) \quad \text { and } \quad \gamma_{8316}=\left(\begin{array}{cccc}
1 & 0 & 0 & 2 \\
-3 & 1 & 2 & -2 \\
-4 & 0 & 1 & -5 \\
0 & 0 & 0 & 1
\end{array}\right)
$$

which fixes $b_{3}^{\prime}$ (respectively $b_{2}^{\prime}$ ) and interchanges $b_{1}^{\prime}$ with $b_{2}^{\prime}$ (respectively with $b_{3}^{\prime}$ ).

The action of $\gamma_{410}$ (respectively $\gamma_{8316}$ ) on $L_{p}^{\prime}$ provides an irreducible polynomial with the same roots as $L_{p}^{\prime}$, which are still in $\mathcal{L}_{p}^{\prime}$ by the following lemma. Hence, this polynomial is $L_{p}^{\prime}$ and thus it is symmetric.

Lemma 43. Let $\Omega \in \mathcal{L}_{p}^{\prime}, \gamma=\left(\begin{array}{ll}A & B \\ C & D\end{array}\right) \in \Gamma_{2} / \Gamma(2,4)$ and $\gamma^{\prime}$ be such that $\gamma_{p}$ is in the same equivalence class of $\gamma^{\prime}$. Suppose that the action of $\gamma^{\prime}$ on the theta constants sends $\{0,4,8,12\}$ to itself. Then $\gamma \Omega$ is in $\mathcal{L}_{p}^{\prime}$.

Proof. For $\Omega \in \Gamma(2,4) \backslash \mathcal{H}_{2}$ to be in $\mathcal{L}_{p}^{\prime}$ means that there exists $M \in \Gamma(2,4) /\left(\Gamma(2,4) \cap \Gamma_{0}(p)\right)$ satisfying $\theta_{0}(p M \Omega / 2)=0$. Let $M^{\prime} \in \Gamma(2,4) /\left(\Gamma(2,4) \cap \Gamma_{0}(p)\right)$ be such that $\left(M^{\prime} \gamma\right) M^{-1} \in \Gamma_{0}(p)$. Then there exists $\gamma^{\prime \prime} \in \Gamma_{0}(p)$ with $M^{\prime} \gamma=\gamma^{\prime \prime} M$. We have, using the duplication formula (Proposition 11)

$$
\theta_{0}\left(p M^{\prime} \gamma \Omega / 2\right)=\theta_{0}\left(p \gamma^{\prime \prime} M \Omega / 2\right)=\theta_{0}\left(\gamma_{p}^{\prime \prime}(p M \Omega) / 2\right)=\sum_{i \in\{0,4,8,12\}} \theta_{i}^{2}\left(\gamma_{p}^{\prime \prime}(p M \Omega)\right)
$$

Moreover, $\gamma_{p}^{\prime \prime}=\left(M^{\prime} \gamma M^{-1}\right)_{p}$ is in the same equivalence class as $\gamma_{p}$, namely $\gamma^{\prime}$ by hypothesis (recall that $\Gamma(2,4)$ is a normal subgroup). The action of $\gamma^{\prime}$ sends $\{0,4,8,12\}$ to itself, so that

$\sum_{i \in\{0,4,8,12\}} \theta_{i}^{2}\left(\gamma_{p}^{\prime \prime}(p M \Omega)\right)=\zeta_{\gamma_{p}^{\prime \prime}}^{2} \operatorname{det}(\ldots) \sum_{i \in\{0,4,8,12\}} \theta_{i}^{2}(p M \Omega)=\zeta_{\gamma_{p}^{\prime \prime}}^{2} \operatorname{det}(\ldots) \theta_{0}(p M \Omega / 2)=0$.

We have proved the following theorem. 
Theorem 44. Let $p>2$ be a prime number. The modular polynomials for $b_{1}^{\prime}, b_{2}^{\prime}$ and $b_{3}^{\prime}$ satisfy

$$
\Phi_{1, p}\left(X, b_{1}^{\prime}, b_{2}^{\prime}, b_{3}^{\prime}\right)=\Phi_{1, p}\left(X, b_{1}^{\prime}, b_{3}^{\prime}, b_{2}^{\prime}\right) \quad \text { and } \quad \Psi_{2, p}\left(X, b_{1}^{\prime}, b_{2}^{\prime}, b_{3}^{\prime}\right)=\Psi_{3, p}\left(X, b_{1}^{\prime}, b_{3}^{\prime}, b_{2}^{\prime}\right)
$$

Morover, the polynomial $L_{p}^{\prime}$ is symmetric.

\subsection{Relations modulo 2 and 4}

Now, we study the different relations modulo 2 and 4 between the exponents of the $b_{i}^{\prime}$ in each coefficient. Consider the numerator of the $\ell$ th coefficient of the $m$ th modular polynomial for $m=1$ or 2 (we have seen that the third polynomial can be deduced from the second one), whose monomials are of the form $c_{i j k} b_{1}^{i} b_{2}^{\prime j} b_{3}^{\prime k}$. We have found that for $p=3,5$ and 7 , if $c_{i j k} \neq 0$, then

$$
\begin{aligned}
i & \equiv \ell+m+1 \bmod 2, \\
i+j & \equiv-p \ell \bmod 4, \\
j+k & \equiv p(m-1) \bmod 4,
\end{aligned}
$$

and with similar notation, we always have

$$
i \equiv j \equiv k \equiv 0 \bmod 2 \text { and } i+j \equiv j+k \equiv 0 \bmod 4
$$

for the denominators. These equalities are determined by the existence of some matrices $\gamma$ with the property that $b_{i}^{\prime}(\gamma \Omega)=\imath^{\alpha_{i}} b_{i}^{\prime}(\Omega)$ and $b_{i, p}^{\prime}(\gamma \Omega)=\imath^{\beta_{i}} b_{i, p}^{\prime}(\Omega)$ with $\alpha_{i}$ and $\beta_{i}$ in $\{0,1,2,3\}$. We will denote the action of such matrices by the vector $\left(\alpha_{1}, \alpha_{2}, \alpha_{3}, \beta_{1}, \beta_{2}, \beta_{3}\right)$.

With the same arguments as before, we deduce that such an action produces a polynomial with the same roots and degree as $\Phi_{1, p}(X)$ (respectively $\Psi_{2, p}(X)$ ) which is then $\Phi_{1, p}(X)$ (respectively $\left.\Psi_{2, p}(X)\right)$ up to a constant. As $p^{3}+p^{2}+p+1 \equiv 0 \bmod 4$ for any prime $p>2$ and as the leading coefficient of $\Phi_{1, p}(X)$ is $X^{p^{3}+p^{2}+p+1}$, we conclude that such an action does not change $\Phi_{1, p}(X)$. This is not the case of $\Psi_{2, p}(X)$ which is of degree $p^{3}+p^{2}+p$ in $X$.

The matrix

$$
\gamma_{134}=\left(\begin{array}{cccc}
-1 & 0 & 0 & 0 \\
0 & -1 & 0 & 0 \\
2 & 1 & -1 & 0 \\
1 & 0 & 0 & -1
\end{array}\right)
$$

acts by $(-1,1,1,-1,1,1)$ for all $p$ by the functional equation of Proposition 8 and Lemma 42 .

Using Lemma 43 shows that this matrix preserves the Humbert component (and $\left(\gamma_{134}\right)_{p}$ is in the same equivalence class of $\gamma_{134}$ by Lemma 42). Thus, we obtain a polynomial with the same roots and degree as $L_{p}^{\prime}$ : it is a multiple of $L_{p}^{\prime}$. As the latter is irreducible, it contains at least one monomial where there is not $b_{1}^{\prime}$ so that the matrix does not change this monomial and the constant is thus 1 . As $L_{p}^{\prime}$ is symmetric, we deduce that it has even exponents in $b_{1}^{\prime}$, $b_{2}^{\prime}$ and $b_{3}^{\prime}$.

If we assume Conjecture 41, then we just proved that the action of $\gamma_{134}$ on the numerators does not depend on its action on the denominator. Then, on the numerators of $\Phi_{1, p}$, the action of $\gamma_{134}$ shows that $i+\ell$ is always even. For $\Psi_{2, p}(X)$, we have to determine the constant which appears. The leading coefficient of this polynomial is $\sum_{\gamma \in C_{p}} b_{2, p}^{\prime \gamma} X^{p^{3}+p^{2}+p}$. Now consider the minimal polynomial $\prod_{\gamma \in C_{p}}\left(X-b_{2, p}^{\prime \gamma}\right)$ of $b_{2}^{\prime}$ and note that it is invariant by the preceding action, 
which is thus also the case for $\sum_{\gamma \in C_{p}} b_{2, p}^{\prime \gamma}$. We deduce that the constant is -1 (because of the $\left.X^{p^{3}+p^{2}+p}\right)$, namely

$$
\sum_{\gamma \in C_{p}} b_{2, p}^{\prime \gamma \gamma_{134}}\left(b_{1, p}^{\prime \gamma_{134}}\right)^{p^{3}+p^{2}+p}=-\sum_{\gamma \in C_{p}} b_{2, p}^{\prime \gamma}\left(b_{1, p}^{\prime}\right)^{p^{3}+p^{2}+p}
$$

We have thus shown the first of the three equalities of (7).

For the other two, we have to consider the matrices

$$
\gamma_{141}=\left(\begin{array}{cccc}
-1 & 0 & 0 & 0 \\
0 & -1 & 0 & 0 \\
1 & 1 & -1 & 0 \\
1 & 1 & 0 & -1
\end{array}\right) \quad \text { and } \quad \gamma_{21}=\left(\begin{array}{cccc}
-1 & 0 & 0 & 0 \\
0 & -1 & 0 & 0 \\
0 & 0 & -1 & 0 \\
0 & 1 & 0 & -1
\end{array}\right) \text {. }
$$

Their action for $p \equiv 1 \bmod 4$ are respectively $(\imath, \imath, 1, \imath, \imath, 1)$ and $(1, \imath, \imath, 1, \imath, \imath)$ and for $p \equiv 3 \bmod 4$ it is $(\imath, \imath, 1,-\imath,-\imath, 1)$ and $(1, \imath, \imath, 1,-\imath,-\imath)$ because in this case $\left(\gamma_{141}\right)_{p}$ and $\left(\gamma_{21}\right)_{p}$ are equivalent to

$$
\gamma_{1886}=\left(\begin{array}{cccc}
-1 & 0 & 0 & 0 \\
0 & -1 & 0 & 0 \\
-1 & 1 & -1 & 0 \\
1 & -1 & 0 & -1
\end{array}\right) \quad \text { and } \quad \gamma_{155}=\left(\begin{array}{cccc}
-1 & 0 & 0 & 0 \\
0 & -1 & 0 & 0 \\
0 & 0 & -1 & 0 \\
0 & 3 & 0 & -1
\end{array}\right) \text {. }
$$

On $L_{p}^{\prime}$, the action of $\gamma_{141}$ does not change the Humbert component by Lemma 43 , so that $L_{p}^{\prime}\left(\imath b_{1}^{\prime}, \imath b_{2}^{\prime}, b_{3}^{\prime}\right)$ is a multiple of $L_{p}^{\prime}$.

As $L_{p}^{\prime}$ is irreducible, there is a monomial without $b_{1}^{\prime}$, which is then of the form $c b_{2}^{\prime i} b_{3}^{\prime j}$ for some constant $c$. We have already shown that $i \equiv j \equiv 0 \bmod 2$ so that $c\left(\imath b_{2}^{\prime}\right)^{i} b_{3}^{\prime j}= \pm c b_{2}^{\prime i} b_{3}^{\prime j}$. If it is equal, then the action of $\gamma_{141}$ fixes $L_{p}^{\prime}$. Otherwise, $i \equiv 2 \bmod 4$ and as $L_{p}^{\prime}$ is symmetric, we also have the monomials $c b_{1}^{i i} b_{2}^{\prime j}$ and $c b_{3}^{i} b_{2}^{\prime j}$. Now look at the latter: $c b_{3}^{\prime i}\left(\imath b_{2}^{\prime}\right)^{j}= \pm c b_{3}^{i} b_{2}^{\prime j}$. If it is not equal, then $j \equiv 2 \bmod 4$ and then $c\left(\imath b_{1}^{\prime}\right)^{i}\left(\imath b_{2}^{\prime}\right)^{j}=c b_{1}^{\prime i} b_{2}^{\prime j}$. In all cases, the action of $\gamma_{141}$ fixes $L_{p}^{\prime}$. We can adapt this proof on $\gamma_{121}$ and deduce (8).

We use similar arguments on $\Phi_{1, p}(X)$ and $\Psi_{\ell, p}(X)$ to prove (7). Thus, we obtain the following theorem.

Theorem 45. Let $p>2$ be a prime number. Then the polynomial $L_{p}^{\prime}$ satisfy (8). Moreover, if we assume Conjecture 41, the numerators of the two first modular polynomials verify (7).

\section{Implementation}

\subsection{External packages}

Dupont presented two algorithms to compute theta functions. The first one uses the definition as sums of exponentials and it computes $\theta_{i}(\Omega)$ for $i=0,1,2,3, \Omega \in \mathcal{F}_{2}$ at precision $N$ with a complexity of $O\left(\mathcal{M}^{\prime}(N) N\right)$. The second one uses Newton lifts and the Borchardt mean and is in $O\left(\mathcal{M}^{\prime}(N) \log (N)\right)$ under Conjecture 23. It computes $\theta_{i}^{2}(\Omega) / \theta_{0}^{2}(\Omega), i=1,2,3$. These algorithms have been studied and implemented by Enge and Thomé in [16, 17]. Using finite differences, they proved that the complexity to compute the squares of the theta constants is in $O\left(\mathcal{M}^{\prime}(N) \log (N)\right)$ under Conjecture 23.

We used the cmh library written in $\mathrm{C}$ for the evaluation of the square of the theta functions (we also recovered from it the implementation of Mestre's algorithm and some other functions that were already written in GP) and we used the pari-gnump software [13] for switching between number types from the GNU multiprecision ecosystem (GMP, MPFR and MPC [14, 20, 22]) and corresponding types in Pari/GP to be able to use the algorithm of cmh with GP.

There are two reasons why the algorithms to compute the theta constants are defined for $\Omega$ only in the fundamental domain. The first one is for the convergence and the second is 
because we can use the functional equation of Proposition 8 to obtain the theta constants at $\Omega \in \mathcal{H}_{2}$ from the theta constants at $\Omega^{\prime} \in \mathcal{F}_{2}$. We have implemented an algorithm to compute the squares of the theta constants for any matrix in $\mathcal{H}_{2}$ with GP [1].

For Algorithm 33, we need a method to reduce some $\Omega \in \mathcal{H}_{2}$ into the fundamental domain. We implemented the standard method (see $[\mathbf{9}, \mathbf{1 9}]$ ). We also used the code of Pascal Molin [33] to compute $\Omega \in \mathcal{H}_{2}$ corresponding to a given hyperelliptic curve equation.

Moreover, we have to know the cosets of $\Gamma(2,4) /\left(\Gamma_{0}(p) \cap \Gamma(2,4)\right)$ for some primes $p$. They are naturally calculated beforehand. A generalization of $[\mathbf{9}$, Algorithm 2] to dimension 2 allows one to compute, for subgroups $\Gamma^{\prime} \subset \Gamma$ of $\Gamma_{2}$, the representatives of the classes of $\Gamma / \Gamma^{\prime}$ and a set of generators of $\Gamma^{\prime}$ from a set of generators of $\Gamma$ and from a function which decides if a matrix lies in $\Gamma^{\prime}$ or not. We apply it twice: first on $\Gamma=\Gamma_{2}$ and $\Gamma^{\prime}=\Gamma(2,4)$, then on $\Gamma=\Gamma(2,4)$ and $\Gamma^{\prime}=\Gamma_{0}(p) \cap \Gamma(2,4)$. Another solution consists in using [9, Proposition 10.1] which provides a set of representatives of $\Gamma_{2} / \Gamma_{0}(p)$ for all $p \geqslant 2$. We have to multiply each representative by a matrix in $\Gamma_{0}(p)$ such that the resulting matrix is in $\Gamma(2,4)$, which is possible by the Chinese remainder theorem.

\subsection{Evaluation and interpolation}

Until now we have presented the algorithm from a theoretical point of view. In practice, we proceed as follows. Since we want to use fast interpolation, it is necessary to know the degrees of the coefficients in the three invariants $f_{1}, f_{2}$ and $f_{3}$. For example, let $F\left(f_{1}, f_{2}, f_{3}\right)$ be one of the coefficients we want to compute. To obtain the total degree of the numerator and of the denominator of $F$, it is enough to compute the matrices, $\Omega$, in the Siegel space with Algorithm 33 such that $\left(f_{1}(\Omega), f_{2}(\Omega), f_{3}(\Omega)\right)=\left(x_{i}, x_{i} y, x_{i} z\right)$ for some $x_{i}$ and fixed $y$ and $z$, to evaluate $F\left(x_{i}, x_{i} y, x_{i} z\right)$ and then to do the interpolation of a univariate rational fraction. This also gives upper bounds for the degrees in $f_{1}, f_{2}$ and $f_{3}$. To obtain the degrees in $f_{1}$ (and similarly in the others), we can compute $F\left(x_{i}, y, z\right)$ and interpolate, but this will not give the a correct answer every time (even if we assume that the precision is correct and that we have enough $x_{i}$ ). Indeed, some simplifications may occur. Thus, to be sure of the result, it is preferable to evaluate and interpolate for many values of $y$ and $z$ and also for $F(X+r, y+s, z+t)$ for some values of $r, s$ and $t$.

Once we have this information, we have two choices for how to proceed. The first entails doing a sufficient number of evaluations to compute all the coefficients (in $X$ ) of the three modular polynomials by interpolation of rational fractions. An evaluation means the computation of the modular polynomials at $\Omega$ such that $\left(f_{1}(\Omega), f_{2}(\Omega), f_{3}(\Omega)\right)$ is of the form $\left(x_{i}, x_{i} y_{j}, x_{i} z_{k}\right)$. Otherwise, we focus first on only one coefficient (the one with the lowest total degree) to compute the common denominator and then we do a sufficient number of evaluations (here of the form $\left.\left(x_{i}, y_{j}, z_{k}\right)\right)$ to compute the other coefficients using interpolation of multivariate polynomials. We can speak about polynomials because we can multiply each evaluation by the evaluation of the denominator (and in the case of the Streng invariants, also by an exponent of $\left.i_{3}\right)$.

In the first case, the number of evaluations will depend on the maximal total degree of the three polynomials, while in the second case, the total degree will intervene only for the coefficient with lowest degree. Moreover, the precision needed to interpolate rational fractions is greater than that needed to interpolate polynomials (and the complexity of an evaluation of the modular polynomials at some matrices of $\mathcal{H}_{2}$ depends on the precision) and it is easier to interpolate polynomials than rational fractions. For the second choice, the degree tables suggest focusing on the coefficient of highest degree (in $X$ ) of $\Phi_{1, p}(X)$.

One can choose to take integer values for the invariants. The matrix $\Omega$ with these invariants and also the invariants of the isogenous varieties will not take integer values, but each coefficient of the evaluated modular polynomials will be a rational number. Thus, it could be possible 
at each evaluation to find these rational numbers using continued fractions (if the working precision is good enough). The interpolation phase could then be done using exact values. However, when doing this, the precision needed will, in practice, increase and the time of evaluation will too. It is preferable to take floating-point values for the invariants and reconstruct the rational numbers once the polynomials have been interpolated at the working precision to find the exact coefficients.

\subsection{Timings}

Note that in the evaluation there are two steps: given $\left(f_{1}(\Omega), f_{2}(\Omega), f_{3}(\Omega)\right)$ find $\Omega$ and then evaluate the modular polynomials at $\Omega$. The latter one takes most of the time (at large enough precision). For example, for $p=5$ and 7 at precision 1000 decimal digits it takes 0.5 seconds to compute $\Omega$ from the $b_{i}^{\prime}(\Omega)$ and the computation of the two polynomials $\Phi_{1, p}\left(X, b_{1}^{\prime}(\Omega), b_{2}^{\prime}(\Omega), b_{3}^{\prime}(\Omega)\right)$ and $\Psi_{2, p}\left(X, b_{1}^{\prime}(\Omega), b_{2}^{\prime}(\Omega), b_{3}^{\prime}(\Omega)\right)$ take 12 and 30 seconds for $p=5$ and $p=7$ respectively (this difference is due to the number of isogenies: 156 for one and 400 for the other).

Now, we focus on the computation of the modular polynomials with Streng invariants (recall the results of $\S 4.1$ ). We proceed with the second method which is not always faster (because it requires two evaluation steps), but it has the advantage of providing the denominator which is the origin of most of the difficulties when computing modular polynomials. Moreover, we do the interpolation of univariate rational fractions with linear algebra because it is fast enough.

In level 2, the largest total degree of the numerator of the coefficient of degree 14 of $\Phi_{1,2}(X)$ is 9 and that of the denominator $D_{2}^{\prime}$ is 7 . To compute the denominator, it is enough to do $(9+7+2)(5+1)(4+1)=540$ evaluations. Once we have computed them, we do $(33+1)(17+$ $1)(16+1)=10404$ evaluations to compute the numerators (see Table 1). All of this can be done at a precision of 100 decimal digits. An evaluation takes around 1.33 second so that the denominator can be computed in around 12 minutes and all the polynomials in 4 hours (on one processor).

In level 3 , the total degree is 35 for both the numerator (of the coefficient of degree 39 of $\left.\Phi_{1,3}\right)$ and the denominator. The denominator can be computed with $(35+35+2)(20+$ 1) $(17+1)=27216$ evaluations in 17 hours at precision 300 and then all the numerators with $(92+1)(52+1)(49+1)=246450$ evaluations (see Table 2$)$ in around 30 days at precision 1000 . (The difference in precision here comes from the fact that the integers of the denominator are much smaller than the integers of the numerators). The interpolation phase takes around 1 hour.

To compute the modular polynomials with the $b_{i}^{\prime}$, we can use the results found in $\S \S 4.2$ and 5. In particular, we only have to compute the first two modular polynomials.

For $p=3$, the total degrees are 25 and 24 for the numerator and the denominator of the 39th coefficient. It takes around $(25+24+2)(12+1)(12+1) / 32 \approx 270$ evaluations to obtain the denominator and around $(40+1)(19+1)(18+1) / 32 \approx 487$ for the numerators (see Table 3$)$. We used 100 decimal digits for the precision and then an evaluation takes approximately 0.6 seconds so that the (two and thus the three) modular polynomials can be obtained in less than 10 minutes (the interpolation phase is negligible).

For $p=5$, the total degrees are 121 and 120 for the numerator and the denominator of the 155 th coefficient. The theoretical number of evaluations for the denominator and the numerator are $(121+120+2)(72+1)(72+1) / 32<40500$ and $(156+1)(97+1)(94+1) / 32<46000$ (see Table 4). They can be done at a precision of 1000 decimal digits where each evaluation takes roughly 12 seconds. The polynomials can be calculated in less than 12 days (on one processor). The interpolation can be done in less than 2 hours.

For $p=7$, we first computed the common denominator because of memory space (the first two polynomials fill 29 GB). Moreover, we found that the leading coefficients of the 
denominators in $b_{1}^{\prime}$ are $2^{10} b_{2}^{\prime 6} b_{3}^{\prime 6} b_{1}^{\prime 10}$ and $2^{70} b_{2}^{\prime 10} b_{3}^{\prime 10} b_{1}^{\prime 70}$ respectively, so we conjectured that it would be of the same kind for $p=7$. Through experimentation, we found that it was $2^{226} b_{2}^{\prime 38} b_{3}^{\prime 38} b_{1}^{\prime 226}$. Knowing this monomial allows one to interpolate, as explained in the second paragraph after Remark 19, which reduces the number of evaluations because this number depends on the degree in $b_{1}^{\prime}$ instead of the total degree.

The degrees of the 399th coefficient are 233 and 226 (and the total degrees are 337 and 336 so that the gain is significant). The number of evaluations for the denominator was around $(233+226+2)(226+1)(226+1) / 32<727000$ and for the numerators of the two modular polynomials around $(400+1)(279+1)(276+1) / 32<972000$ (see Table 4$)$. For the denominator, we managed to compute it in less than 700 days at precision 2000 and for the numerators in around 2000 days at precision 3000. The interpolation time was around a week. It is negligible compared to the evaluation time.

Finally note that each evaluation is independent of the others so that the computation of modular polynomials is highly parallelizable. The interpolation of a coefficient is independent of the interpolation of the others so that the interpolation step is also parallelizable. Moreover, it is possible to parallelize the interpolation of a single coefficient.

\section{Examples of isogenous curves}

The main purpose of the modular polynomials is to find hyperelliptic curves with isogenous Jacobians, in particular over a finite field. We give some examples with the different polynomials we have computed. Note that the algorithm we have presented is heuristic because we have no bounds on the precision loss and we have no proof that the polynomials we found are correct. We could do interval arithmetic: what we do instead is to heuristically check for correctness on additional random values not yet used during the evaluation/interpolation algorithm. For some $\Omega \in \mathcal{H}_{2}$, we have to verify that

$$
\Phi_{1, p}\left(f_{1, p}(\Omega), f_{1}(\Omega), f_{2}(\Omega), f_{3}(\Omega)\right)=0
$$

and that for $\ell=2,3$

$$
f_{\ell, p}(\Omega)=\Psi_{\ell, p}\left(f_{1, p}(\Omega), f_{1}(\Omega), f_{2}(\Omega), f_{3}(\Omega)\right) / \Phi_{1, p}^{\prime}\left(f_{1, p}(\Omega), f_{1}(\Omega), f_{2}(\Omega), f_{3}(\Omega)\right) .
$$

With one high precision computation, one can be virtually certain that the result is correct.

The Jacobians of the following curves are $(3,3)$-isogenous varieties. We computed the curves using the modular polynomials with Streng invariants. The first ones over $\mathbb{F}_{5261}$ are

$$
\begin{aligned}
& Y^{2}=272 X^{5}+4278 X^{4}+4297 X^{3}+4063 X^{2}+1069 X+2998, \\
& Y^{2}=695 X^{5}+2322 X^{4}+3115 X^{3}+4588 X^{2}+1453 X+655
\end{aligned}
$$

and the ones over $\mathbb{F}_{2534267893}$ are

$$
\begin{aligned}
Y^{2}= & 1774507961 X^{6}+48872812 X^{5}+2028583210 X^{4}+1092030439 X^{3} \\
& +671225738 X^{2}+2233670825 X+608155867, \\
Y^{2}= & 1927466494 X^{6}+2286039407 X^{5}+1720123333 X^{4}+87910848 X^{3} \\
& +2422852850 X^{2}+183139891 X+825611194 .
\end{aligned}
$$

We also give two examples of curves with $(5,5)$-isogenous Jacobians computed using the modular polynomials with the $b_{i}^{\prime}$. Over $\mathbb{F}_{101}$ we have

$$
\begin{aligned}
& Y^{2}=27 X^{5}+71 X^{4}+91 X^{3}+59 X^{2}+5 X+14, \\
& Y^{2}=29 X^{5}+26 X^{4}+38 X^{3}+20 X^{2}+7 X+51
\end{aligned}
$$

and over $\mathbb{F}_{4294967311}$ we have

$$
\begin{aligned}
Y^{2}= & 2420332800 X^{5}+3653091983 X^{4}+2536585478 X^{3}+2805510580 X^{2} \\
& +159741347 X+2690010753, \\
Y^{2}= & 4076826784 X^{5}+2616936853 X^{4}+3748957676 X^{3}+1209100179 X^{2} \\
& +3172892980 X+1266950302 .
\end{aligned}
$$


Finally, we give two pairs of curves with $(7,7)$-isogenous Jacobians, computed using the modular polynomials with the $b_{i}^{\prime}$. Over $\mathbb{F}_{10009}$ we have

$$
\begin{aligned}
& Y^{2}=4826 X^{5}+471 X^{4}+2876 X^{3}+5411 X^{2}+7948 X+1308 \\
& Y^{2}=7218 X^{5}+7699 X^{4}+7011 X^{3}+7103 X^{2}+1845 X+4087
\end{aligned}
$$

and over $\mathbb{F}_{3452678353}$ we have

$$
\begin{aligned}
Y^{2}= & 393356368 X^{5}+1698662093 X^{4}+471351782 X^{3}+448279016 X^{2} \\
& +1342046779 X+3241061457 \\
Y^{2}= & 2171506943 X^{5}+2231412358 X^{4}+2005208933 X^{3}+580698082 X^{2} \\
& +306153493 X+474327543 .
\end{aligned}
$$

The motivated reader can check the curves we have constructed with the modular polynomials: it is enough to verify that the curves have the same zeta functions.

The polynomials are accessible at the address: http://www.math.u-bordeaux1.fr/ emilio/.

Acknowledgements. Experiments presented in this paper were carried out using the PLAFRIM experimental testbed, being developed under the Inria PlaFRIM development action with support from LABRI and IMB and other entities: Conseil Régional d'Aquitaine, FeDER, Université de Bordeaux and CNRS (see https://plafrim.bordeaux.inria.fr/). The author thanks his PhD supervisors, Andreas Enge and Damien Robert, not only for the fruitful discussions we had, but also for their support and encouragement during his studies. The author also thanks the anonymous reviewer for his careful reading and his comments and suggestions.

\section{References}

1. K. Belabas et al., PARI/GP version 2.5.3, Bordeaux, 2012, http://pari.math.u-bordeaux.fr/.

2. J. Belding, R. Bröker, A. Enge and K. Lauter, 'Computing Hilbert class polynomials', Algorithmic Number Theory 8th International Symposium (ANTS VIII), Lecture Notes in Computer Science 5011 (Springer, Berlin, 2008) 282-295.

3. C. Birkenhake and H. Lange, Complex abelian varieties, Grundlehren der Mathematischen Wissenschaften 302 (Springer, 2003).

4. G. Bisson and A.V. Sutherland, 'Computing the endomorphism ring of an ordinary elliptic curve over a finite field', J. Number Theory 113 (2011) 815-831.

5. R. Bröker and K. Lauter, 'Modular polynomials for genus 2', LMS J. Comput. Math. 12 (2009) no. 1, 326-339.

6. R. Bröker, K. Lauter and A. V. Sutherland, 'Modular polynomials via isogeny volcanoes', Math. Comp. 81 (2012) 1201-1231.

7. R. Cosset, 'Applications des fonctions thêta à la cryptographie sur courbes hyperelliptiques', PhD Thesis, Université Henri Poincaré - Nancy 1, 2011.

8. P. Davis and P. Rabinowitz, Methods of Numerical Integration, 2nd edn (Academic Press, New York, 1984).

9. R. Dupont, 'Moyenne arithmético-géométrique, suites de Borchardt et applications', PhD Thesis, École polytechnique, 2006, http://www.lix.polytechnique.fr/Labo/Regis.Dupont/.

10. K. Eisenträger and K. LAuter, 'A CRT algorithm for constructing genus 2 curves over finite fields', Arithmetic, Geometry and Coding Theory (AGCT-10), Séminaires et Congrès 21 (Société Mathématique de France, Paris, 2009) 161-176.

11. N. Elkies, 'Elliptic and modular curves over finite fields and related computational issues', Computational perspectives on number theory: Proceedings of the conference in honor of A.O.L. Atkin, AMS/IP Studies in Advanced Mathematics 7 (American Mathematical Society, Boston, 1998) 21-76.

12. A. Enge, 'Computing modular polynomials in quasi-linear time', Math. Comp. 78 (2009) 1809-1824, http://hal.inria.fr/hal-00823745.

13. A. EnGE, PARI-GNUMP, version 0.0.1, February 2014, http://www.multiprecision.org/index.php?prog= pari-gnump/.

14. A. Enge, M. Gastineau, P. Théveny and P. Zimmermann, GnU MPC - a library for multiprecision complex arithmetic with exact rounding, version 1.0.1, INRIA, September 2012, http://mpc.multiprecisi on.org/. 
15. A. Enge and A. V. Sutherland, 'Class invariants by the CRT method', Algorithmic Number Theory 9th International Symposium (ANTS IX), Lecture Notes in Computer Science 6197 (Springer, Berlin, 2010) $142-156$.

16. A. Enge and E. Thomé, 'Computing class polynomials for abelian surfaces', Exp. Math. 23 (2014) 129-145.

17. A. Enge and E. Thomé., CMH - computation of Igusa class polynomials, version 1.0, March 2014, http://cmh.gforge.inria.fr/.

18. E. Freitag, Siegelsche Modulfunktionen, Grundlehren der Mathematischen Wissenschaften 254 (Springer, Berlin, 1983).

19. E. Gottschling, 'Explizite Bestimmung der Randflächen des Fundamentalbereiches der Modulgruppe zweiten Grades', Ann. of Math. (2) 138 (1959) 103-124.

20. T Granlund et al., GMP - the GNU multiple precision arithmetic library, version 5.1.1, February 2013, http://gmplib.org/.

21. D. Gruenewhld, 'Explicit algorithms for Humbert surfaces', PhD Thesis, University of Sydney, 2008.

22. G. Hanrot, V. Lefìvre, P. PÉlissier and P. Zimmermann et al., GNU MPFR - a library for multipleprecision floating-point computations with exact rounding, version 3.1.1, July 2012, http://www.mpfr.org/.

23. F. Hirzebruch and G. VAn Der Geer, Lectures on Hilbert modular surfaces, Séminaire de Mathématiques Supérieures 77 (Presses de l'université de Montréal, Montréal, 1981).

24. J. van Der Hoeven, 'Fast evaluation of holonomic functions', Theoret. Comput. Sci. 210 (1999) no. 1, $199-215$.

25. J. von zur Gathen and G. Jürgen, Modern Computer Algebra (Cambridge University Press, New York, NY, 1999).

26. J. I. IGusA, 'Arithmetic variety of moduli for genus 2', Ann. of Math. (2) 72 (1960) no. 3, 612-649.

27. J. I. Igusa, 'On Siegel modular forms of genus 2', Amer. J. Math. 84 (1962) 175-200.

28. J. I. Igusa, Theta functions, Grundlehren der Mathematischen Wissenschaften 194 (Springer, Berlin, 1972).

29. H. KLIngen, Introductory lectures on Siegel modular forms, Cambridge Studies in Advanced Mathematics 20 (Cambridge University Press, Cambridge, 1990).

30. S. LANG, Elliptic functions, Graduate Text in Mathematics 112 (Springer, New York, NY, 1987).

31. R. Manni, 'Modular varieties with level 2 theta structure', Amer. J. Math. 116 (1994) 1489-1511.

32. J.-F. Mestre, 'Construction de courbes de genre 2 à partir de leurs modules', Effective methods in algebraic geometry, Progress in Mathematics 94 (Birkhäuser, Boston, MA, 1991) 313-334.

33. P. Molin, 'Intégration numérique et calculs de fonctions $L$ ', $\mathrm{PhD}$ Thesis, Université Bordeaux 1, 2010. https://github.com/pascalmolin/hcperiods.

34. D. Mumford, Abelian Varieties, Tata Institute of fundamental research studies in mathematics (Published for the Tata Institute of Fundamental Research, Bombay by Oxford University Press, 1970).

35. D. Mumford, Tata lectures on theta I, Progress in Mathematics 28 (Birkhäuser, Boston, MA, 1983).

36. D. Mumford, Tata lectures on theta II, Progress in Mathematics 43 (Birkhäuser, Boston, MA, 1984).

37. B. Runge, 'Endomorphism rings of abelian surfaces and projective models of their moduli spaces', Tohoku Math. J. (2) 51 (1999) no. 3, 283-303.

38. R. Schertz, Complex multiplication, New Mathematical Monographs 15 (Cambridge University Press, New York, NY, 2010).

39. L. SCHLÄFLI, 'Beweis der Hermiteschen Verwandlungstafeln für die elliptischen Modulfunktionen', J. Reine Angew. Math. 72 (1870) 360-369.

40. R. Schoof, 'Counting points on elliptic curves over finite fields', J. Théor. Nombres Bordeaux 7 (1995) 219-264.

41. A. Schönhage and V. Strassen, 'Schnelle Multiplikation grosser Zahlen', Computing 7 (1971) 281-292.

42. M. Streng, 'Complex multiplication of abelian surfaces', PhD Thesis, Universiteit Leiden, 2010.

43. A. V. Sutherland, 'Computing Hilbert class polynomials with the Chinese remainder theorem', Math. Comp. 80 (2011) 501-538.

44. J. Thомаe, 'Beitrag zur Bestimmung von $\theta(0,0, \ldots, 0)$ durch die Klassenmoduln algebraischer Funktionen', J. Reine Angew. Math. 70 (1870) 201-222.

45. A. Weng, 'Constructing hyperelliptic curves of genus 2 suitable for cryptography', Math. Comp. 72 (2003) no. $241,435-458$.

46. Don ZAGIER, Modular forms of one variable. Notes based on a course given in Utrecht, 1991. http://people.mpim-bonn.mpg.de/zagier/files/tex/UtrechtLectures/UtBook.pdf.

\section{Enea Milio}

\section{INRIA Bordeaux Sud-Ouest}

33405 Talence

France

enea.milio@inria.fr 